DOI: $10.24143 / 2072-9502-2020-4-80-100$

UDC 511.1:004.056

\title{
PRIME NUMBER LAW. DEPENDENCE OF PRIME NUMBERS ON THEIR ORDINAL NUMBERS AND GOLDBACH - EULER BINARY PROBLEM USING COMPUTER
}

\author{
S. I. Chermidov
}

Kuban State University,

Krasnodar, Russian Federation

\begin{abstract}
The article considers the methods of defining and finding the distribution of composite numbers $C N$, prime numbers $P N$, twins of prime numbers $T w$ and twins of composite numbers $T w C N$ that do not have divisors 2 and 3 in the set of natural numbers - $\mathbb{N}$ based on a set of numbers like $\Theta=\{6 \cdot \kappa \pm 1, \kappa \in \mathbb{N}\}$, which is a semigroup in relation to multiplication. There has been proposed a method of obtaining primes $p \geq 5$ by using their ordinal numbers in the set of primes $p \geq 5$ and vice versa, as well as a new algorithm for searching and distributing primes based on a closedness of the elements of the set $\Theta$. It has been shown that a composite number $n \in \Theta$ can be presented in the form of products $(6 x \pm 1)(6 y \pm 1)$, where $x, y \in \mathbb{N}$ - are positive integer solutions of one of the 4 Diophantine equations: $P(x, y, \lambda)=6 x y \pm x \pm y-\lambda=0$. It has been proved that if there is a parameter $\lambda$ of prime twins, then none of Diophantine equations $P(x, y, \lambda)=0$ has positive integer solutions. There has been found the new distribution law of prime numbers $\pi(x)$ in the segment $[1 \div N]$. Any even number $\zeta>8$ is comparable to one of the numbers $m=(0,2,-2)$, i.e. $\zeta \equiv m(\bmod 6)$. According to the above remainders $m$, even numbers $\zeta>8$ are divided into 3 types, each type having its own way of representing sums of 2 elements of the set $\Theta$. For any even number $\zeta>8$ in a segment $[1 \div v]$, where $v=(\zeta-\mathrm{m}) / 6, v=(\zeta-m) / 6$, there is a parameter of an even number; it is proved that there is always a pair of numbers $\left(\lambda_{1}, \lambda_{2}\right) \in[1 \div v]$ that are elements of the united sets of parameters of prime twins $\Pi_{T w}$ and parameters of transition numbers $\Pi_{U P C}$, i.e. numbers of the form $6 \lambda \pm 1$ with the same $\lambda$, if the form $6 \lambda-1$ is a prime number, then the form $6 \lambda+1$ is a composite number, and vice versa.
\end{abstract}

Key words: prime and composite numbers, parameters of primes, Diophantine equations, binary (strong) Goldbach - Euler, algorithm for solving the binary Goldbach - Euler problem.

For citation: Chermidov S. I. Prime number law. Dependence of prime numbers on their ordinal numbers and Goldbach - Euler binary problem using computer. Vestnik of Astrakhan State Technical University. Series: Management, Computer Science and Informatics. 2020;4:80-100. (In Russ.) DOI: 10.24143/2072-9502-2020-4-80-100.

\section{Introduction}

Gauss was the first who observed the regularity of the arrangement of primes showing that the probability of the appearance of primes in the int $(1 \div x)$ is equal to $\frac{x}{\ln x}$. Then Legendre improved the type $\Lambda(x)=\frac{x}{\ln x-1.08366}$ and introduced the function $\pi(x)$. The first essential contribution to the study of $\pi(x)$ was made by Euler with his $\zeta(s)$-function, [1] with the real variables $s$, $\zeta(s)=\sum_{n=1}^{\infty} \frac{1}{n^{s}}=\prod_{p}\left(1-\frac{1}{p^{s}}\right),^{-1} s>1$. By means of it, we showed that the series $\sum_{p} p^{-1}$ diverge, which means that primes are infinite. In 1859 Riemann proposed to consider the variation of the variable $s$ of the Euler function in the complex plane and related the distribution of primes to the nontrivial zeros of the $\zeta(s)$ - function B. Riemann formulated the problem now known as the Riemann hypothesis, name- 
ly, that all the nontrivial zeros of the $\zeta(s)$ - function are in the strip $0 \leq \operatorname{Re} s \leq 1$ and are symmetric with respect to the $\zeta(s)$ critical line $\frac{1}{2}+i x$, where $x \in R$, i. e. all nontrivial zeros of $\zeta(s)$ having a real part $\frac{1}{2}$ are complex numbers. In the work of $V$. Minayev an attempt was made to describe the laws of natural numbers, on the basis of the elements of the set $\Theta$ with the introduction of concepts of fundamental negative n-s $\theta^{-}=6 \kappa-1$, positive $n-s \theta^{+}=6 \kappa+1$, composite $n$-s \& primes. But nothing is said about the methods and definitions, i.e. at what values of the parameters $\kappa$, the numbers $\theta^{-} \& \theta^{+}$ are composite or prime. In 2013, Itan Chang proved that there are infinitely many pairs of successive primes with a difference $\leq 7 \cdot 10^{7}$ and Terens Tao reduced this diffeerence to 246 . In recent decades interest in the laws of prime distribution [1] from the theoretical point of view has been increasingly shifting to the practical one. Their use in cryptography is a particularly important example [2], that is why any results that clarify certain features of the laws of prime distribution immediately become the subject of study of the field cryptographers. Special interest in cryptography in a system with public keys (in particular, in the RSA encryption system) raises the question, whether this particular (large) number is prime or not. Interest in Goldbach - Euler binary problem in mathematics, as well as its application in related sciences and technologies are very important in cryptography [1,2]. In the last decade the significant advances have been made in the field of additive number theory. For example, in 2013 Harald Helfgott proved Goldbach ternary problem. From the properties of even number $\zeta>8$, we can see that are $\zeta \equiv m(\bmod 6)$, where $m=(0,2,-2)$ are considered and their representations as sums of 2 numbers of the form $\theta_{1}=6 \lambda_{1} \pm 1$ and $\theta_{2}=6 \lambda_{2} \pm 1$. An even number $\zeta>8$ in the segment $\Lambda_{1}=\left[1 \div\left[\frac{v}{2}\right]\right]$ and $\Lambda_{2}=\left[\left[\frac{v}{2}\right] \div v\right]$, where $v=\frac{\zeta-m}{6}$, is an even number parameter. It has been proved that there always exist a pair of numbers $\lambda_{1} \in \Lambda_{1}$ and $\lambda_{2} \in \Lambda_{2}$, where $\left(\lambda_{1}, \lambda_{2}\right) \in\left(\Pi_{T w} \cup \Pi_{U P C}\right)$. The

aim of the paper is to study the laws of prime distribution and composite numbers $C N$, to prove the solvability of the Diophantine equation $z=x+y$ in the domain of integers $Z^{+}, z>2$ for any even number and the summands $x, y \in P$.

\section{Method for selecting prime numbers}

When searching for primes in a natural series, a large loss of time is necessary for numbers that are divisible by $2 \& 3$. If in the set $\mathbb{N}$, there is a factorization with base 6 , we can simplify the search for primes and study their properties. We divide the set $\mathbb{N}$ into 2 and disjoint subsets $H$ and $\Theta i$.

$\mathrm{H} \cap \Theta=\varnothing, \mathbb{N}=\mathrm{H} \cup \Theta$. Let $H$ include 1 (one) and natural numbers, which after division by 6 give the remainders $0,2,3,4$. Obviously, the set $H$ includes two primes 2 and 3. The set $\Theta$ contains numbers which when divided by 6 give the remainders 1 and 5, i.e. numbers of the form, $\Theta=\{6 \kappa \pm 1, \kappa \in \mathbb{N}\}$, as in the expression $6 m+5=6(m+1)-1$. Numbers with reminders $0,2,3,4$ are composite numbers, since they are divisible by 2 or 3 . So, we have embedding the sets $P(\geq 5) \subset \Theta \subset \mathbb{N}$, where $P(\geq 5)$ is a set of primes $p \geq 5$. It is not difficult to verify directly that the elements of a set $\Theta=\{6 \cdot \kappa \pm 1, \kappa \in \mathbb{N}\}$, are closed under the operation of multiplication, i.e. the set $\Theta$ is a semigroup with operation of multiplicationumbers. We have relations:

$$
\begin{array}{ll}
\text { 1. } n=(6 x-1)(6 y-1)=6(6 x y-x-y)+1 . & 3 . n=(6 x+1)(6 y-1)=6(6 x y-x+y)-1 . \\
\text { 2. } n=(6 x+1)(6 y+1)=6(6 x y+x+y)+1 . & 4 . n=(6 x-1)(6 y+1)=6(6 x y+x-y)-1 .
\end{array}
$$

Products of numbers of the forms $\theta_{1}=6 x \pm 1$ and $\theta_{2}=6 y \pm 1$ are expressed by numbers of the form, $6 x y \pm x \pm y$ and it is obvious that the secret of composite and prime numbers lies here. Algorithms and programs of obtaining and distributing primes and composite numbers, twins of primes and twins of composite numbers were performed using Computer Software and Computing. 
Definition. For a number $n$, the values of the numerical functions $\lambda(x, y)=6 x y \pm x \pm y$ $\forall x, y \in \mathbb{N}$ represented in (1) will be called the parameters of the number $n \in \Theta$. We note that the problem of a single - valued correspondence between the numbers $\mathrm{n}$ and their parameters $\lambda(x, y)$ remains open, i. e several different parametric functions $\lambda(x, y)$ can correspond to the same number $n$. However, from the point of view of later studies, this fact is not significant. We note that the form $6 \lambda-1$ can be transformed into another form $6 \lambda+1$ by multiplication (1). Since the set $\Theta$ is a semigroup with respect to the operation of multiplication, all its constituent elements $\forall \lambda_{\mathrm{i}} \in \mathbb{N}$ will be:

$$
\theta=\left(6 \lambda_{1} \pm 1\right) \cdot\left(6 \lambda_{2} \pm 1\right) \cdot\left(6 \lambda_{3} \pm 1\right) \cdot \ldots \cdot\left(6 \lambda_{i} \pm 1\right) .
$$

In the set $\Theta$ there are elements $\theta=6 \lambda \pm 1$, for which the number of factors in (2) is equal to 1 , i. e. they do not decompose into products of other numbers in $\Theta$, these numbers are primitive elements of the set $\Theta$, i. e., primes $p \geq 5$ in the set $\mathbb{N}$. For each of the expressions (1) we introduce a parameter $\lambda=6 x y \pm x \pm y$, then from the link (1) we have:

$$
\lambda=\frac{(n \pm 1)}{6}
$$

where the "-" sign corresponds to the first two expressions (1) and the "+" sign - to the last two expressions (1). That is, we obtain equations $P(x, y, \lambda)=0$ for finding positive integer $Z^{+}$connecting the numbers $(x, y)$ and parameter $\lambda$ :
1. $6 x y-x-y-\lambda=0, \quad P_{1}(x, y, \lambda)=0$.
3. $6 x y-x+y-\lambda=0, \quad P_{3}(x, y, \lambda)=0$.
2. $6 x y+x+y-\lambda=0, \quad P_{2}(x, y, \lambda)=0$.
4. $6 x y+x-y-\lambda=0, \quad P_{4}(x, y, \lambda)=0$.

If at least one of the equations in (4) has one solution, then the number $n=6 \lambda \pm 1$ from (1) is a composite number. If for some $\lambda$ the Diophantine equations from (4) do not have positive integer solutions, then the number $n$ from (1) for this $\lambda$ is a prime number. It is quite obvious from representations (1) and (2) that any constituent element $\theta=(6 \lambda \pm 1) \in \Theta$ will ultimately disintegrate into a product of primitive elements. Whence the main theorem of arithmetic follows, that any positive integer numbers $n>1$ can be represented as a product: $n=p_{1}^{d_{1}}, \cdot p_{2}^{d_{2}}, \ldots, \cdot p_{k}^{d_{k}}$, where $p_{1}, p_{2}, \ldots, p_{k}$ are primes and $d_{1}, d_{2}, \ldots, d_{k}$ are the numbers of corresponding primes involved in the decompositionumbers.

\section{The distribution of composite numbers of the set $\Theta$}

From (1) one can see that the primes and composite numbers are formed by values of the following functions:

$$
\begin{array}{lll}
\text { 1. } \quad \lambda_{1}=f_{11}(x, y)=6 x y-x-y . & \text { 3. } \quad \lambda_{3}=f_{21}(x, y)=6 x y-x+y . \\
\text { 2. } \quad \lambda_{2}=f_{12}(x, y)=6 x y+x+y . & \text { 4. } \quad \lambda_{4}=f_{22}(x, y)=6 x y+x-y,
\end{array}
$$

- composite numbers of the set $\Theta$ of the form $6 \lambda+1$ (we denote them $C N^{+}$) in view of (1) are generated by values of the symmetric and non-one-to-one functions: $f_{11}(x, y)=6 x y-x-y$, $f_{12}(x, y)=6 x y+x+y$, since for unequal values $\left(x_{1}, y_{1}\right) \neq\left(x_{2}, y_{2}\right)$ the values of the functions can be equal to $f_{11}\left(x_{1}, y_{1}\right)=f_{11}\left(x_{2}, y_{2}\right)$ and $f_{12}\left(x_{1}, y_{1}\right)=f_{12}\left(x_{2}, y_{2}\right)$. With allowance for (1) we notice that the numbers of the forms $\theta_{1}=6 \lambda_{1}+1$ and $\theta_{2}=6 \lambda_{2}+1$ are composite numbers $\theta_{1}, \theta_{2} \in C N^{+}$;

- composite numbers of set $\Theta$ of the form $6 \lambda-1$ (we denote them $C N^{-}$) in view of (1) are generated by the values of the non-symmetric and non-one-to-one functions $f_{21}(x, y)=6 x y-x+y \& f_{22}(x, y)=6 x y+x-y$. It also follows from (1) that the numbers of forms $\theta_{3}=6 \lambda_{3}-1$ and $\theta_{4}=6 \lambda_{4}-1$ are composite numbers, $\theta_{3}, \theta_{4} \in C N^{-}$. Since the numbers $\theta_{1}, \theta_{2}$ are 
the composite numbers, then the values of the variables $(x, y)$ are solutions of the corresponding equations $P_{1}(x, y, \lambda)=0$ or $P_{2}(x, y, \lambda)=0$. And in the same way, for composite numbers $\theta_{3}, \theta_{4}$ values of $(x, y)$ are solutions of the equations $P_{3}(x, y, \lambda)=0$ or $P_{4}(x, y, \lambda)=0$. We also note that for the factorization (taking into account that prime number is the least divisor [3]) of a composite number, the most effective and the best way is to use expression (2) that is the number $n \in \Theta$ divided by numbers of the form $6 \lambda \pm 1$, where $\lambda=1,2,3, \ldots$ (see [4]). From (4) it is easy to deduce that the set of parameters of all 4 types of primes and composite numbers of set $\Theta$ in the natural series of numbers is infinite. Indeed, for example, if the range of function $f_{11}(x, y)$ value from (5) is defined as the set $\Phi_{x, y}=\{6 x y-x-y\}$, then for any specific number $y=n \in \mathbb{N}, x \rightarrow \infty$ the expression $\{(6 n-1) x-n\} \rightarrow \infty$.

Similarly, other functions are also considered as $f_{i, j}(x, y), 1 \leq i, j \leq 2$ given in (5), we denote the sets as $G_{1}=\left\{f_{i, j}(x, y) \mid x=1, y \geq 1\right\}, G_{2}=\left\{f_{i, j}(x, y) \mid x=2, y \geq 2\right\}, G_{v}=\left\{f_{i, j}(x, y) \mid x=v, y \geq v\right\}, v \in Z^{+}$.

Thus, the set of values of all the functions (5) in Table 1: $G=G_{1} \cup G_{2} \cup \ldots \cup G_{\mathrm{v}}$ are infinite as unions of infinite sets. We introduce the notation for the set of parameters of composite numbers of the forms:

$$
\left\{\begin{array}{l}
\text { 1. } 6 \lambda+1: \lambda \in F N^{+}=f_{11}(x, y) \cup f_{12}(x, y) . \\
\text { 2. } 6 \lambda-1: \lambda \in F N^{-}=f_{21}(x, y) \cup f_{22}(x, y) .
\end{array}\right.
$$

Thus, if all composite numbers of the set $\Theta$ consist of a union $C N=C N^{-} \cup C N^{+}$or $C N=\left\{6 \lambda-1\right.$, where $\left.\lambda \in F N^{-}\right\} \cup\left\{6 \lambda+1\right.$, where $\left.\lambda \in F N^{+}\right\}$, then the set of all parameters of the composite numbers $\Theta$ will be a union $F N=F N^{-} \cup F N^{+}$. It is obvious that the sets $F N, F N^{+}, F N^{-}$are infinite as unions of infinite sets. To determine and study the parameters of primes and composite numbers of the set $\Theta$, it will be necessary to find all the parametric solutions of the equations (4). However, the solution of Diophantine equations is a complicated problem, therefore, to solve equations (4) we can construct a table of values of the function $\lambda(x, y)$ or functions (5). Then to the number $\lambda$ in the table there corresponds a composite number $n$, otherwise the number $n$ is prime numbers. To study the parameters of primes and composite numbers of the set $\Theta$, we set any values $x, y \in \mathbb{N}$ for the values of the functions (5) from 1 to $s \in \mathbb{N}$, where $s$ is the specified table size. Let us construct Table 1 in the dimension $s \times s$. Note that for the same values of the variables $x, y \in \mathbb{N}$ in each row $(x, y)$ of Table 1 we have an increasing sequence of functions:

$$
f_{11}(x, y)<f_{22}(x, y) \leq f_{21}(x, y)<f_{12}(x, y) .
$$

Forming strings $(x, y)$ in Table 1 and the search for the values of the functions (5) are carried out, according to the principle:

$$
\int_{\text {from } x=1 \text { from }}^{t o s} \int_{y=x}^{t o s} f_{11}(x, y), f_{12}(x, y), f_{21}(x, y), f_{22}(x, y)
$$

We choose the value $s=10$ to demonstrate the algorithms described below, but the constructions described below can be realized for any $s$. Let us find the values of the functions: $f_{11}(x, y), f_{12}(x, y), f_{21}(x, y), f_{22}(x, y)$, where $6 \cdot f_{11}(x, y)+1 ; 6 \cdot f_{12}(x, y)+1 ; 6 \cdot f_{21}(x, y)+1$ $6 \cdot f_{22}(x, y)+1$ is composite number, since the values of the variables $(x, y)$ are known as predetermined solutions of the equations (4). Let $x \in \mathbb{N}$, then the values of the functions (5) in the following row $(x, y+1)$ differ from the values of the previous row $(x, y)$ to the following value functions: for function $f_{11}(x, y)$ by $6 x-1$, for function $f_{12}(x, y)$ by $6 x+1 \&$ for function $f_{21}(x, y)$ by $6 x+1$, 
for function $f_{22}(x, y)$ by $6 x-1$. Let $m_{1}=f_{11}(x, y+1)-f_{11}(x, y)=6 x-1 ; \quad m_{2}=f_{22}(x, y+1)-$ $-f_{22}(x, y)=6 x-1 \quad m_{3}=f_{21}(x, y+1)-f_{21}(x, y)=6 x+1 ; \quad m_{4}=f_{12}(x, y+1)-f_{12}(x, y)=6 x+1$.

Since $\forall x \in Z^{+}$the differences $\left(m_{1}, m_{2}, m_{3}, m_{4}\right)>0$, then the functions (5) are increasing and infinite.

Example 1. Find the composite numbers of the set $\Theta$ in the int $1 \div N=155$. We calculate in the specified interval the max parameter: $\lambda_{\max }=\left[\frac{N}{6}\right]+1=26$, from the Table 1 we write the parameters of composite numbers $\leq \lambda_{\text {max }}$. As a result, we have: $\Pi_{C N}=\left\{{ }^{+} 4,{ }^{-} 6,{ }^{+} 8,{ }^{+} 9,{ }^{-} 11,{ }^{-} 13,{ }^{+} 14,{ }^{+} 15,{ }^{-} 16,{ }^{+} 19,{ }^{+}-20\right.$ $\left.-21,{ }^{+} 22,{ }^{+-} 24,-26\right\}$. Symbols in numbers are obtained, according to sets $F N^{+}$and $F N^{-}$. On the basis of the definition of the parameters of the composite numbers from the set $\Theta$ we find their values: $C N^{+}: 6 \cdot 4+1=25 ; 6 \cdot 8+1=49 ; 6 \cdot 9+1=55 ; 6 \cdot 14+1=85 ; 6 \cdot 15+1=91 ; 6 \cdot 19+1=115 ; 6 \cdot 20$ $+1=121 ; 6 \cdot 22+1=133 ; 6 \cdot 24+1=145$.

$C N^{-}: 6 \cdot 6-1=35 ; 6 \cdot 11-1=65 ; 6 \cdot 13-1=77 ; 6 \cdot 16-1=95 ; 6 \cdot 20-1=119 ; 6 \cdot 21-1=125 ;$ $6 \cdot 24-1=143 ; 6 \cdot 26-1=155$. Hence, the complete sequence of composite numbers of $\Theta$ : $C N=C N^{+} \cup C N^{-}=\{25,35,49,55,65,77,85,91,95,115,119,121,125,133,143,145,155\}$.

Table 1

Formation of the parameters of composite numbers in the set $\Theta$

\begin{tabular}{|c|c|c|c|c|c|c|}
\hline $\boldsymbol{G}$ & $\boldsymbol{x}$ & $\boldsymbol{y}$ & $\boldsymbol{f}_{\mathbf{1 1}}(\boldsymbol{x}, \boldsymbol{y})$ & $\boldsymbol{f}_{\mathbf{1 2}}(\boldsymbol{x}, \boldsymbol{y})$ & $\boldsymbol{f}_{\mathbf{2 1}}(\boldsymbol{x}, \boldsymbol{y})$ & $\boldsymbol{f}_{\mathbf{2 2}}(\boldsymbol{x}, \boldsymbol{y})$ \\
\hline$G \mathbf{1}$ & 1 & 1 & 4 & 8 & & 6 \\
\hline & & 2 & 9 & 15 & 13 & 11 \\
\hline & & 3 & 14 & 22 & 20 & 16 \\
\hline & & 4 & 19 & 29 & 27 & 21 \\
\hline & & 5 & 24 & 36 & 34 & 26 \\
\hline & & 6 & 29 & 43 & 41 & 31 \\
\hline & & 7 & 34 & 50 & 48 & 36 \\
\hline & & 8 & 39 & 57 & 55 & 41 \\
\hline & & 9 & 44 & 64 & 62 & 46 \\
\hline & & 10 & 49 & 71 & 69 & 51 \\
\hline$G \mathbf{2}$ & 2 & 2 & 20 & 28 & 24 & 24 \\
\hline & & 3 & 31 & 41 & 37 & 35 \\
\hline & & 4 & 42 & 54 & 50 & 46 \\
\hline & & 5 & 53 & 67 & 63 & 57 \\
\hline & & 6 & 64 & 80 & 76 & 68 \\
\hline & & 7 & 75 & 93 & 89 & 79 \\
\hline & & 8 & 86 & 106 & 102 & 90 \\
\hline & & 9 & 97 & 119 & 115 & 101 \\
\hline & & 10 & 108 & 132 & 128 & 112 \\
\hline$G \mathbf{3}$ & 3 & 3 & 48 & 60 & 54 & 54 \\
\hline & & 4 & 65 & 79 & 73 & 71 \\
\hline & & 5 & 82 & 98 & 92 & 88 \\
\hline & & 6 & 99 & 117 & 111 & 105 \\
\hline & & 7 & 116 & 136 & 130 & 122 \\
\hline & & 8 & 133 & 155 & 149 & 139 \\
\hline & & 9 & 150 & 174 & 168 & 156 \\
\hline & & 10 & 167 & 193 & 187 & 173 \\
\hline$\rightarrow$ & & & & & & $\rightarrow$ \\
\hline & & & & & \\
\hline & & & & \\
\hline
\end{tabular}

\begin{tabular}{|c|c|c|c|c|c|c|}
\hline $\boldsymbol{G}$ & $\boldsymbol{x}$ & $\boldsymbol{y}$ & $\boldsymbol{f}_{\mathbf{1 1}}(\boldsymbol{x}, \boldsymbol{y})$ & $\boldsymbol{f}_{\mathbf{1 2}}(\boldsymbol{x}, \boldsymbol{y})$ & $\boldsymbol{f}_{\mathbf{2 1}}(\boldsymbol{x}, \boldsymbol{y})$ & $\boldsymbol{f}_{\mathbf{2 2}}(\boldsymbol{x}, \boldsymbol{y})$ \\
\hline$G_{\mathbf{4}}$ & 4 & 4 & 88 & 104 & 96 & 96 \\
\hline & & 5 & 111 & 129 & 121 & 119 \\
\hline & & 6 & 134 & 154 & 146 & 142 \\
\hline & & 7 & 157 & 179 & 171 & 165 \\
\hline & & 8 & 180 & 204 & 196 & 188 \\
\hline & & 9 & 203 & 229 & 221 & 211 \\
\hline & & 10 & 226 & 254 & 246 & 234 \\
\hline $\boldsymbol{G}_{\mathbf{5}}$ & 5 & 5 & 140 & 160 & 150 & 150 \\
\hline & & 6 & 169 & 191 & 181 & 179 \\
\hline & & 7 & 198 & 222 & 212 & 208 \\
\hline & & 8 & 227 & 253 & 243 & 237 \\
\hline & & 9 & 256 & 284 & 274 & 266 \\
\hline & & 10 & 285 & 315 & 305 & 295 \\
\hline$G 6$ & 6 & 6 & 204 & 228 & 216 & 216 \\
\hline & & 7 & 239 & 265 & 253 & 251 \\
\hline & & 8 & 274 & 302 & 290 & 286 \\
\hline & & 9 & 309 & 339 & 327 & 321 \\
\hline & & 10 & 344 & 376 & 364 & 356 \\
\hline$G 7$ & 7 & 7 & 280 & 308 & 294 & 294 \\
\hline & & 8 & 321 & 351 & 337 & 335 \\
\hline & & 9 & 362 & 394 & 380 & 376 \\
\hline & & 10 & 403 & 437 & 423 & 417 \\
\hline$G \mathbf{8}$ & 8 & 8 & 368 & 400 & 384 & 384 \\
\hline & & 9 & 415 & 449 & 433 & 431 \\
\hline & & 10 & 462 & 498 & 482 & 478 \\
\hline$G \mathbf{9}$ & 9 & 9 & 468 & 504 & 486 & 486 \\
\hline & & 10 & 521 & 559 & 541 & 539 \\
\hline$G \mathbf{1 0}$ & 10 & 10 & 580 & 620 & 600 & 600 \\
\hline & & & & & & \\
\hline & & & & \\
\hline
\end{tabular}

Distribution of the parameters of primes and composite numbers $\Theta$ in $\mathbb{N}$

The distribution of the parameters of primes and composite numbers of the set $\Theta$ is the analog of the distribution of primes $p \geq 5$ and composite numbers that do not have divisors 2 and 3 in $\mathbb{N}$. It will be necessary to find all primes $\Theta$ of the form $6 \lambda \pm 1$. We describe the algorithms for constructing these numbers. Let in the int $[1 \div n)$ the entries in the file are given according to the structure 
$R_{\pi}=\left(i d .\left[F_{1}\right] \cdot\left[F_{2}\right]\right)$, where the field $i d$ are the serial numbers of the records and the fields $F_{1}$ and $F_{2}$ take the values "+" or "-". Before the beginning of algorithms 1 and 2 below in the interval $[1 \div[n / 6])$ the sign "+" are entered line by line in the fields $F_{1}$ and $F_{2}$ [5].

1. Algorithm distributing primes of a set $\Theta$ of the form $6 \lambda-1$. Let $(x, y)=1,2,3, \ldots$ vary according to the principle of Table 1 . Then, by values of the parameters $\lambda=6 x y-x-y$ and $\lambda=6 x y+x+y$ of a composite number of the form $6 \lambda+1 \in \Theta$ from a file $R_{\pi}$ by direct access to the records $i d=\lambda$ in the field $F_{2}$ the sign "+" changes into the sign "--" and the remaining records at the end of the algorithm in the adjacent field $F_{1}=$ "+" indicate the presence of primes of type: $6 \lambda-1 \in P N^{-}$. We introduce the notation $\Xi_{11}=\bigcup_{x, y}^{\infty} f_{11}(x, y) \Xi_{12}=\bigcup_{x, y}^{\infty} f_{12}(x, y), K_{-1}=\Xi_{11} \bigcup \Xi_{12}$, then $P N^{-}=\left\{6 \lambda-1, \lambda \in K_{-1}\right\}$.

2. Algorithm distributing primes of a set $\Theta$ of the form $6 \lambda+1$. Let $(x, y)=1,2,3, \ldots$ vary according to the principle of Table 1 . Then by values of the parameters $\lambda=6 x y-x+y$ and $\lambda=6 x y+x-y$ of composite numbers of the form $6 \lambda-1 \in \Theta$ from a file $R_{\pi}$ by direct access to the records $i d=\lambda$ in the field $F_{1}$ the sign "+" changes to the sign "--" and the remaining records at the end of the algorithm in adjacent field $F_{2}=$ " + " indicate the presence of primes of $6 \lambda+1 \in P N^{+}$.

We introduce the notation $\Xi_{21}=\bigcup_{x, y}^{\infty} f_{21}(x, y) ; \Xi_{22}=\bigcup_{x, y}^{\infty} f_{22}(x, y) ; K_{+1}=\Xi_{21} \quad \bigcup \Xi_{22}$, then $P N^{+}=\left\{6 \lambda+1, \lambda \in K_{+1}\right\}$.

So, the primes consist of the union of two sets: $P=P N^{-} \cup P N^{+}$. Combining algorithms 1 and 2 into one algorithm [5] we obtain the algorithm for distributing parameters of composite numbers and primes $\Theta$ in $\mathbb{N}$. Parameters $i d=\lambda$ with the assigned fields $F_{1}$ and $F_{2}$ with the value "+", according algorithms to 1 and 2, are parameters of twins of primes and with the value "-" are the parameters of twins of composite numbers.

Theorem 1. At least one of the equations (4) has solutions when the form $6 \lambda+1$ or $6 \lambda-1$ is composite number.

Necessity. Let a number of the form $n=6 \lambda+1$ is a composite number, since $\Theta$ is a semigroup, where exist 2 numbers $\theta_{1}=6 x_{0} \pm 1$ and $\theta_{2}=6 y_{0} \pm 1, \theta_{1}, \theta_{2} \in \Theta$ with $\left(x_{0}, y_{0}\right) \in \mathbb{N}$, as $n=6 \lambda+1=\left(6 x_{0} \pm 1\right)\left(6 y_{0} \pm 1\right)=6\left(x_{0} y_{0} \pm x_{0} \pm y_{0}\right) \pm 1$. According to (1), there are only 2 cases for numbers, i. e. $\theta_{1}=6 x_{0}-1 ; \theta_{2}=6 y_{0}-1$, which gives $\lambda=6 x_{0} y_{0}-x_{0}-y_{0}$ and $\theta_{1}=6 x_{0}+1 ; \theta_{2}=6 y_{0}+1$ which gives $\lambda=6 x_{0} y_{0}+x_{0}+y_{0}$. In any case, one of the 2 first equations (4) has positive solutionumbers. The same is true for numbers of the form $n=6 \lambda-1$, in which case one of the two last equations (4) has positive solutionumbers. This completes the proof.

Adequacy. Suppose, one of the equations (4), for example, equation $6 x y-x-y-\lambda=0$, has a solutionumbers. This means that there exists a triple of natural numbers $(\lambda, x, y) \in \mathbb{N}$, that is fair $\lambda=6 x y-x-y$. Then from $P_{1}(x, y, \lambda)=0$ we have $n=6(6 x y-x-y)+1=(6 x-1)(6 y-1)$, i. e. the number corresponding to parameter $\lambda$ is a composite number, since both of $(6 x-1)(6 y-1)$ are natural numbers different from 1 , because $(x, y) \neq 0$. The same is true for any of the equations (4).

Solutions of Diophantine equations $\boldsymbol{P}(x, y, \lambda)=\mathbf{0}$

Example 2.

2.1. We know some solution of one of the equations (4). 
1. Let $\left(\begin{array}{l}x=11 \\ y=2\end{array}\right.$, are solution of $P_{1}(x, y, \lambda)=0 \rightarrow \lambda=6 x y-x-y=119$. Taking (3) into account, we have $\frac{N-1}{6}=119$ or $n=6 \cdot 119+1=715$. From $(1) \rightarrow 715=(6 \cdot 11-1)(6 \cdot 2-1) \rightarrow n=715$ is a composite $n$.

2. Let $\left(\begin{array}{l}x=2 \\ y=5\end{array}\right.$, is a solution of $P_{3}(\lambda, 2,5)=0$, which $\lambda=6 \cdot 2 \cdot 5-2+5=63$. Taking (3) into account, we have $\frac{n+1}{6}=63$ from $(1) \rightarrow 377=(6 \cdot 2+1)(6 \cdot 5-1)$, i. e $n=6 \cdot 63-1$ is a composite numbers.

2.2. We know that for some $\lambda$ one of the corresponding numbers is prime.

1. Let $=16 \rightarrow n=6 \cdot 16+1=97 \in P$, hence, $P_{1}(x, y, \lambda)=0$ and $P_{2}(x, y, \lambda)=0$ have no solutions.

2. Let $\lambda=15 \rightarrow n=6 \cdot 15-1=89 \in P$, hence, $P_{3}(x, y, \lambda)=0 \& P_{4}(x, y, \lambda)=0$ have no solutions.

2.3. We know that for some parameter $\lambda$ one of the corresponding numbs is composite. Taking $\lambda=7589$, we know that $n=6 \cdot 7589+1=5 \cdot 9107$ is a composite number. Hence, one of the Diophant equations $P_{1}(x, y, \lambda)$ or $P_{2}(x, y, \lambda)$ has solutions.

1. $6 x y-x-y=7589$. If we express $y$ in terms of $x$, than $y=\frac{7589+x}{6 x-1}$, which must be an integer. So, we search for which values of $x$ in $1 \div(\lambda-1)$ the above value of $y$ is an integer. Easily found $\left(\lambda, x_{1}, y_{1}\right)=(7589,1,1518),\left(\lambda, x_{2}, y_{2}\right)=(7589,6,217), \quad\left(\lambda, x_{3}, y_{3}\right)=(7589,217,6),\left(\lambda, x_{4}, y_{4}\right)=$ $=(7589,1518,1)$.

2. $6 x y+x+y=7589$. If we express $y$ in terms of $x$, we have $y=\frac{7589-x}{6 x+1}$, which must be an integer. So, we search for which values of $x$ in $1 \div(\lambda-1)$ the above value of $y$ is an integer. We found 2 solutions $\left(\lambda, x_{1}, y_{1}\right)=(7589,1,1084),\left(\lambda, x_{2}, y_{2}\right)=(7589,1084,1)$. Let $\lambda=63$, we know that $n=6 \cdot 63-1=377=13 \cdot 29$ is a composite numbers Hence, one of $P_{3}(x, y, \lambda)=0$ or $P_{4}(x, y, \lambda)=0$ has solutions.

3. $6 x y-x+y=63$. We rewrite last relation as $y=\frac{63+x}{6 x+1}$, which must be an integer. So, we search for which values of $x$ in $1 \div(\lambda-1)$ the above of $y$ is an integer. One solution of the triplet $(2,5,63)$ is found.

4. $6 x y+x-y=63$. We express $y$ in terms of $x$, and have $y=\frac{63-x}{6 x-1}$, which must be an integer. So, we search for which values of $x$ in $1 \div(\lambda-1)$ the above of $y$ is an integer. One solution $(2,5,63)$ is found. According to the distribution of the parameters of prime and composite numbers without divisors 2 and 3, we compose a Table 2 .

Table 2

Distribution of the parameters of primes and $\mathrm{CN}$ of the set $\Theta$ in the $\mathbb{N} *$

\begin{tabular}{|c|c|c|c|c|c|c|c|c|c|c|c|c|c|c|}
\hline$I d=\lambda$ & $F_{1}$ & $F_{2}$ & $C$ & $C$ & 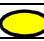 & $P$ & 3 & $C$ & $P$ & 3 & 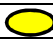 & $P$ & 3 & $\infty$ \\
\hline 1 & + & + & 41 & - & - & 81 & - & + & 121 & - & + & 161 & - & + \\
\hline 2 & + & + & 42 & + & - & 82 & + & - & 122 & - & + & 162 & + & - \\
\hline 3 & + & + & 43 & + & - & 83 & - & + & 123 & - & + & 163 & + & - \\
\hline 4 & + & - & 44 & + & - & 84 & + & - & 124 & + & - & 164 & + & - \\
\hline 5 & + & + & 45 & + & + & 85 & + & - & 125 & - & + & 165 & - & + \\
\hline 6 & - & + & 46 & - & + & 86 & - & - & 126 & - & + & 166 & - & + \\
\hline 7 & + & + & 47 & + & & 87 & + & + & 127 & + & - & 167 & - & - \\
\hline 8 & + & - & 48 & - & - & 88 & - & - & 128 & - & + & 168 & - & + \\
\hline
\end{tabular}


Distribution of the parameters of primes and $\mathrm{CN}$ of the set $\Theta$ in the $\mathbb{N}^{*}$

\begin{tabular}{|c|c|c|c|c|c|c|c|c|c|c|c|c|c|c|}
\hline$I d=\lambda$ & $F_{1}$ & $F_{2}$ & & 8 & $\odot$ & & 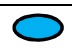 & $C$ & & & & & & $C$ \\
\hline 9 & + & - & 49 & + & - & 89 & - & - & 129 & + & - & 169 & + & - \\
\hline 10 & + & + & 50 & & - & 90 & - & + & 130 & - & - & 170 & + & + \\
\hline 11 & - & + & 51 & - & + & 91 & - & + & 131 & - & + & 171 & - & - \\
\hline 12 & + & + & 52 & + & + & 92 & - & - & 132 & - & - & 172 & + & + \\
\hline 13 & - & + & 53 & + & - & 93 & +100 & - & 133 & + & - & 173 & - & + \\
\hline 14 & + & - & 54 & - & - & 94 & + & - & 134 & - & - & 174 & - & - \\
\hline 15 & + & - & 55 & - & + & & + & + & 135 & + & + & 175 & + & + \\
\hline 16 & - & + & 56 & - & + & 96 & - & + & & - & - & 176 & - & - \\
\hline 17 & + & + & 57 & - & - & 97 & - & - & 137 & + & + & 177 & + & + \\
\hline 18 & + & + & 58 & + & + & 98 & + & - & 138 & + & + & 178 & - & + \\
\hline 19 & + & - & 59 & + & - & 99 & + & - & 139 & - & - & 179 & - & - \\
\hline 20 & - & - & 60 & + & - & 100 & + & + & 140 & + & - & 180 & - & - \\
\hline 21 & - & + & 61 & - & + & 101 & - & + & 141 & - & - & 181 & - & + \\
\hline 22 & + & - & 62 & - & + & 102 & - & + & 142 & - & + & 182 & + & + \\
\hline 23 & + & + & 63 & - & + & 103 & + & + & 143 & + & + & 183 & + & - \\
\hline 24 & - & - & 64 & + & - & 104 & - & - & 144 & + & - & 184 & + & - \\
\hline 25 & + & + & 65 & + & - & 105 & - & + & 145 & - & - & 185 & + & - \\
\hline 26 & - & + & 66 & - & + & 106 & - & - & 146 & - & + & 186 & - & + \\
\hline 27 & - & + & 67 & + & - & 107 & + & + & 147 & +150 & + & 187 & - & + \\
\hline 28 & + & - & 68 & - & + & 108 & + & - & 148 & + & - & 188 & - & + \\
\hline 29 & + & - & 69 & - & - & 109 & + & - & 149 & - & - & 189 & - & - \\
\hline 30 & + & + & 70 & + & + & 110 & + & + & 150 & - & - & 190 & - & - \\
\hline 31 & - & - & 71 & - & - & 111 & - & - & 151 & - & + & 191 & - & - \\
\hline 32 & + & + & 72 & + & + & 112 & - & + & 152 & + & - & 192 & + & + \\
\hline 33 & + & + & 73 & - & + & 113 & + & - & 153 & - & + & 193 & - & - \\
\hline 34 & - & - & 74 & + & - & 114 & + & - & 154 & - & - & 194 & + & - \\
\hline 35 & - & + & 75 & + & - & 115 & - & + & 155 & + & - & 195 & - & + \\
\hline 36 & - & - & 76 & - & + & 116 & - & - & 156 & - & + & 196 & - & - \\
\hline 37 & - & + & 77 & + & + & 117 & + & - & 157 & + & - & 197 & + & - \\
\hline 38 & + & + & 78 & + & - & 118 & - & + & 158 & + & - & 198 & + & - \\
\hline 39 & + & - & 79 & - & - & 119 & - & - & 159 & + & - & 199 & + & - \\
\hline 40 & +50 & + & 80 & + & - & 120 & + & - & 160 & - & - & 200 & - & + \\
\hline
\end{tabular}

*The values of elements of the set $\Theta$, respectively, over the fields $F_{1}: 6 \lambda-1$ and $F_{2}: 6 \lambda+1$. By analogy to the filds $F_{1}, F_{2}$, the "+" sign corresponds to primes and "-" to composite numbers. It is obvious that these subsets of numbers in the natural series are parameters of the corresponding subsets of the set $\Theta$.

Ascribing to serial numbers of records signs "+" or "-", in the fields $F_{1}$ and $F_{2}$ form a table of signs (Table 2) and using algorithms 1 and 2, the natural numbers are divided into subsets of numbers according to the combinations of the signs "+" \& "-". Consider the relationship between the equations (4) and the twins of primes. From the definition of twins of primes it is known that these are the $p_{1}, p_{2} \in P$ with $\left|p_{2}-p_{1}\right|=2$. Note that for the same $\lambda$ the difference of $\theta_{\lambda}^{+}-\theta_{\lambda}^{-}=2$. So, if for this value of the $\lambda$ the numbers $\theta_{\lambda}^{+} \in P N^{+} \& \theta_{\lambda}^{-} \in P N^{-}$are primes, then the pair of these numbers is generating a twin of primes. Hence, for this value of $\lambda$ the numbers $6 \lambda \pm 1$ will be twin primes.

Theorem 2. In order that $\lambda \in \mathbb{N}$ is a parameter of twins of primes, it is necessary and sufficient that for this particular $\lambda$ none of the Diofantine equations (4) has solutions.

Necessity. Suppose for one and the same $\lambda \in \mathbb{N}$ all equations (4) have no solutions. Then, by Theorem 1 from equations $P_{1}(x, y, \lambda)$ and $P_{2}(x, y, \lambda)$ it follows that $\theta_{\lambda}^{+}=6 \lambda+1$ is a prime number and from equations $P_{3}(x, y, \lambda)$ and $P_{4}(x, y, \lambda)$ it follows that $\theta_{\lambda}^{-}=6 \lambda-1$ is a prime number. Since $\theta_{\lambda}^{+}-\theta_{\lambda}^{-}=2$, then, by definition, $\theta_{\lambda}^{+}, \theta_{\lambda}^{-}$of twins of primes and this $\lambda$ is a parameter of twins of primes.

Adequacy. Let $\lambda$ be a parameter of twins of primes, i. e. $p_{2}=6 \lambda+1, p_{1}=6 \lambda-1$ are primes by virtue of the definition of the twins of primes $p_{2}-p_{1}=2, p_{1}, p_{2} \in P$. Hence, from Corollary 1 , for 
a prime number $p_{2}=6 \lambda+1$ it follows that the equations $P_{1}(x, y, \lambda)=0 \& P_{2}(x, y, \lambda)=0$ have no solutions and from Corollary 2 the equations $P_{3}(x, y, \lambda)=0 \& P_{4}(x, y, \lambda)=0$ it follows that they have no solutions for prime $p_{1}=6 \lambda-1$. Thus, none of the Diophantine equations (4) has solutions.

\section{Definitions of subsets of the set $\Theta$}

By virtue of the distribution of the parameters of primes and composite numbers of the set $\Theta$ in $\mathbb{N}$ the serial numbers $i d=\lambda$ (see Table 2), which are parameters of the following significant subsets ( $T w$ - twins of primes), (TwCN - twins of composite numbers do not have divisors 2 and 3 ), ( $U C$ - unique composite numbers), $(P N-$ unique primes) and $(U P C=U C \cup P N)$, of union unique primes and unique composite numbers).

Twin composite numbers ( 2 composites numbers on the forms $6 \lambda \pm 1$ with the same parameter $\lambda$, having a difference $=2)$, i. e. $T w C N=\left\{6 \lambda \pm 1, \lambda \in \Pi_{T w C N}\right\}$, where

$$
\Pi_{T w C N}=F N^{-} \cap F N^{+} .
$$

The set $\Pi_{T w C N}$ is parameters $\lambda$ of twins of composite numbers, which lie on non - empty intersections of the values of two functions (5), one of which belongs to $F N^{+}$and the other to $F N^{-}$. The values of the fields $F_{1}=$ " - " and $F_{2}=$ " $-"$ in Table 2 correspond to the parameters $\lambda$, the set of twins of composite numbers.

2. Unique composite numbers (the difference between 2 composite numbers without divisors 2 and 3 is $>2$

$$
U C=\left\{6 \lambda-1: \lambda \in F N^{-} \backslash \prod_{T w C N}\right\} \cup\left\{6 \lambda+1: \lambda \in F N^{+} \backslash \prod_{T w C N}\right\},
$$

where $F N^{-}$from (6) is the set of parameters $\lambda$ that are representable in the form $\{6 x y-x+y\}$ or $\{6 x y+x-y\}$ for some values $(x, y) \in \mathbb{N}$ and $F N^{+}$, is the set of parameters $\lambda$ that are representable in the form $\{6 x y-x-y\}$ or $\{6 x y+x+y\}$. The parameters of unique composite numbers $\Pi_{U C}=\boldsymbol{F} N^{\top} \backslash \Pi_{T w C N} \cup F N^{+} \backslash \Pi_{T w C N}=\left(F N^{+} \cup F N\right) \backslash\left(F N^{+} \cap F N\right)=F N^{+} \Delta F N^{\top}$ - we have the symmetric difference of sets

Obviously, the parameters of all composite numbers will be $C N=U C \cup T w C N$ and $\rightarrow \Pi_{C N}=\Pi_{U C} \cup \Pi_{T w C N}$.

3. Unique primes

$$
P N=(2 \& 3) \cup\left\{6 \lambda+1, \lambda \in F N^{-} \backslash \Pi_{T w C N} \cup 6 \lambda-1, \lambda \in F N^{+} \backslash \Pi_{T w C N}\right\} .
$$

Parameters $\Pi_{P N}=F N^{-} \backslash \Pi_{T w C N} \cup F N^{+} \backslash \Pi_{T w C N}=\left(F N^{+} \cup F N^{-}\right) \backslash\left(F N^{+} \cup F N^{-}\right)=F N^{+} \Delta F N^{-}$i. e. the symmetric difference of sets. Since the set of parameters $F N^{-} \backslash \prod_{T w C N}$ are not solutions of equations $P_{1}(x, y, \lambda)=0, P_{2}(x, y, \lambda)=0$, then by Corollary 1 number of the form $6 \lambda+1$ is a prime number and set of parameters $F N^{+} \backslash \Pi_{T w C N}$ are not solutions of equations $P_{3}(x, y, \lambda)=0, \quad P_{4}(x, y, \lambda)=0$, then by Corollary 2 number of the form $6 \lambda-1$ is prime.

4. Twins of primes (part 2): $T w=\left\{6 \lambda \pm 1, \lambda \in \Pi_{T w}\right\}$, where

$$
6 \lambda-1 \in P N^{-} ; 6 \lambda+1 \in P N^{+} .
$$

Parameters of twins of primes are at the intersections of the complements of the sets $P N^{-} \&$ $P N^{+}$i. e. $\Pi_{\mathrm{T} w} \subset \mathbb{N} \backslash F N$. In Table 2 the parameters of twins of primes $\lambda$ correspond to the values of the fields $\left(F_{1}=\right.$ "+"; $F_{2}=$ "“"). Then, the parameters of all primes $\Pi_{P}=\Pi_{P N} \cup \Pi_{T w}$.

5. Transition numbers (Unique Prime Composite)

$$
U P C=\left\{6 \lambda \pm 1, \lambda \in F N^{+} \Delta F N^{-}\right\}
$$


That is, numbers go from composite numbers to primes or vice versa by changing the forms $6 \lambda+1 \& 6 \lambda-1$ with the same parameter $\lambda$. The parameters $\Pi_{U P C}=\Pi_{U C} \cup \Pi_{P N}$ corresponded in Table 2 to the values of the fields $\left(F_{1}=\right.$ "-”, $F_{2}=$ “+”) or $\left(F_{1}=\right.$ “+”, $F_{2}=$ " -", $)$.

Lemma 1. The set of natural series of numbers $\mathbb{N}$ and the set $\Theta$ are equivalent sets.

Proof. For two sets $\mathbb{N} \sim \Theta$ to be equivalent, it is necessary and sufficient that there is a one-to-one correspondence between their elements. Note that the distribution of the parameters of the elements of the set $\Theta$ by the design of Table 2 sets to each ordinal number $i d=\lambda$ an element from the subsets $T w C N, T w$, and $U P C$. This means that the elements of set natural series can be fully described by the function (5), i. e. set $\Theta$ covers set $\mathbb{N}$. Hence, we have the right to assert that $\forall n \in \mathbb{N}$ is a parameter of one of the listed subsets of the set $\Theta$. So, it holds: $\mathbb{N}=\Pi_{T w} \cup \Pi_{T w C N} \cup \Pi_{U P C}$.

Lemma 2. Parameters $\lambda_{1}, \lambda_{2}$ and $\lambda_{3}, \lambda_{4}$, respectively, of the unique composite numbers of the types $6 \lambda+1$ and $6 \lambda-1$, then the parameters of unique primes will be numbers of the form $6 \lambda-1$ and $6 \lambda+1$, respectively.

Proof. Excluding the values of parameters $\Pi_{T w C N}$ from Table 1, we have the remaining values of parameters $\lambda_{1}, \lambda_{2}$ of the composite numbers of the form $6 \lambda+1$ and values of parameters $\lambda_{3}, \lambda_{4}$ for composite numbers of the form $6 \lambda-1$. Since the values of parameters $\lambda_{3}, \lambda_{4}$ are in most cases different from the parameters $\lambda_{1}, \lambda_{2}$, (because functions (5) are all different from each other), then, by Corollaries 1-2 they are the parameters of unique primes for the form $6 \lambda+1$ and likewise the values of the parameters $\lambda_{1}, \lambda_{2}$ are not representable with the parameterss $\lambda_{3}, \lambda_{4}$ i. e. are the parameters of unique primes for the form $6 \lambda-1$. So, the laws of the distribution of unique primes by $6 \lambda+1,6 \lambda-1$ are the same as for composite numbers, respectively by type $6 \lambda-1$ and $6 \lambda+1$. And, since the $\lambda_{1}, \lambda_{2}$ and $\lambda_{3}, \lambda_{4}$ are the infinity, then the infinity of unique primes follows from this.

\section{Calculation of primes $p \geq 5$ by their ordinal numbers \& vice versa in the set primes $\boldsymbol{P}$}

From Table 2 of distribution of parameters of primes \& composite numbers of $\Theta$ in $\mathbb{N}$ it is not difficult to see that between the ordinal numbers of primes in the set of primes $P(\geq 5)$ and parameters $\lambda$ of primes $p \geq 5$ shown in Table 2, there are dependencies. Let $n$ be the ordinal number of the prime number $p \geq 5$. in the set of primes $P(\geq 5)$, then the corresponding prime number is found from the formula: $P_{n}=6 \cdot i d+(-1)^{\psi(n)}$, where $i d$ is the line number, $\psi(n)$ is the index of field $F_{\psi(n)}$, on which the $n$-th symbol "+" in Table 2. Counting of the signs "+" is carried out according to the following principle of viewing the lines. At the beginning of the algorithm, the values of the variables $S_{1}=S_{2}=\psi(n)=0$.

a. Algorithm for obtaining a prime by its ordinal number in the prime number $P$.

$$
\begin{gathered}
n \rightarrow P . \quad \sum_{i d=1}^{\text {End of fail }} S_{1}=S_{1}+\left\{\begin{array}{l}
0, F_{1}=“-” \\
1, F_{1}=“+”
\end{array}\right\}_{i d} S_{1}+S_{2}=n_{\mathrm{Yes}, \psi(n)=\downarrow_{\downarrow}}^{?} \text { no } \rightarrow \\
\rightarrow S_{2}=S_{2}+\left\{\begin{array}{l}
0, F_{2}=“-” \\
1, F_{2}=“+”
\end{array}\right\}_{i d} \quad S_{1}+S_{2}=n_{\mathrm{Yes}, \psi(n)=2 \downarrow}^{?}
\end{gathered}
$$

\section{B. Algorithm for obtaining serial numbers by their primes.}

$$
\begin{aligned}
P \rightarrow n, \quad m= & {\left[\frac{p \pm 1}{6}\right]+1, \sum_{i=1}^{m} S_{1}=S_{1}+\left\{\begin{array}{ll}
0, & F_{1}=“-” \\
1, & F_{1}=“+"
\end{array}\right\}_{i} 6 i-1={ }^{?} p_{\text {Yes: } n=S_{1}+S_{2} \downarrow} \text { no } \rightarrow } \\
& \rightarrow S_{2}=S_{2}+\left\{\begin{array}{l}
0, F_{2}=“-” \\
1, F_{2}=“+"
\end{array}\right\} 6 \cdot i+1=? p_{i} p_{\text {Yes, } n=S_{1}+S_{2} \downarrow}
\end{aligned}
$$

Example 3. Let the ordinal number $\boldsymbol{n}=\mathbf{1 0}$ of a prime number be $p \geq 5$, then from Table 2 in the fields $F_{1}$ and $F_{2}$, summing up the number of characters "+" starting from the first line $i d=1$ and on, 
each time we chek the sum $S_{1}+S_{2}$ for equality with $n$. If equal, the algorithm ends, otherwise continues. If equality occurs when calculating $S_{1}$, then $\psi(n)=1$, otherwise for $S_{2}$ then $\psi(n)=2$.

Table 3

Algorithmic way to get a prime number by its ordinal number in the $P(\geq 5)$

\begin{tabular}{|c|c|c|c|c|c|c|}
\hline id & $\begin{aligned} S_{1}= & S_{1}+\left(0: F_{2}="-"\right), \\
& \left(1: F_{2}=\text { "*") }\right)\end{aligned}$ & $\begin{array}{c}S_{1}+S_{2}=n \\
?\end{array}$ & $\psi(n)$ & $\begin{aligned} S_{2}= & S_{2}+\left(0: F_{2}=" 6 "\right) \\
& \left(1: F_{2}=\text { "6+") }\right.\end{aligned}$ & $\begin{array}{c}S_{1}+S_{2}=n \\
?\end{array}$ & $\psi(n)$ \\
\hline 1 & $0+1=1$ & $1+0=1$ & 0 & $0+1=1$ & $1+1=2$ & 0 \\
\hline 2 & $1+1=2$ & $2+1=3$ & 0 & $1+1=2$ & $2+2=4$ & 0 \\
\hline 3 & $2+1=3$ & $3+2=5$ & 0 & $2+1=3$ & $3+3=6$ & 0 \\
\hline 4 & $3+1=4$ & $4+3=7$ & 0 & $3+0=3$ & $4+3=7$ & 0 \\
\hline 5 & $4+1=5$ & $5+3=8$ & 0 & $3+1=4$ & $5+4=9$ & 0 \\
\hline 6 & $5+0=5$ & $5+4=9$ & 0 & $4+1=5$ & $5+5=10$ & 2 \\
\hline
\end{tabular}

Hence, this prime number with the ordinal number $n=10$ in the table of primes $P \geq 5$ will be the number $P_{10}=6 \cdot i d+(-1)^{\psi(n)}=6 \cdot 6+(-1)^{2}=37$. Now, consider the opposite case. Let given a prime number $P=37$, find its serial number $n$.

Table 4

Algorithmic way of getting a serial number by the value of its prime in the $P(\geq 5)$

\begin{tabular}{|c|c|c|c|c|c|c|}
\hline$i$ & $\begin{aligned} S_{1}= & S_{1}+\left(0: F_{1}="-"\right) \\
& \left(1: F_{1}="+"\right)\end{aligned}$ & $n=S_{1}+S_{2}$ & $\begin{array}{c}6 i-1=p \\
?\end{array}$ & $\begin{aligned} S_{2}= & S_{2}+\left(0: F_{2}=\text { "_"” }\right) \\
& \left(1: F_{2}=\text { "*" }\right)\end{aligned}$ & $n=S_{2}+S_{1}$ & $\begin{array}{c}6 . i+1=p \\
?\end{array}$ \\
\hline 1 & $0+1=1$ & $1+0=1$ & $5 \neq p$ & $0+1=1$ & $1+1=2$ & $7 \neq p$ \\
\hline 2 & $1+1=2$ & $2+1=3$ & $11 \neq p$ & $1+1=2$ & $2+2=4$ & $13 \neq p$ \\
\hline 3 & $2+1=3$ & $3+2=5$ & $17 \neq p$ & $2+1=3$ & $3+3=6$ & $19 \neq p$ \\
\hline 4 & $3+1=4$ & $4+3=7$ & $23 \neq p$ & $3+0=3$ & $3+4=7$ & $25 \neq p$ \\
\hline 5 & $4+1=5$ & $5+3=8$ & $29 \neq p$ & $3+1=4$ & $4+5=9$ & $31 \neq p$ \\
\hline 6 & $5+0=5$ & $5+4=9$ & $35 \neq p$ & $4+1=5$ & $5+5=10$ & $P=\mathbf{3 7}$ \\
\hline
\end{tabular}

\section{Primes distribution and formula for finding $\pi(x)$}

For the calculation of primes in $(1 \div x)$ there are functions of 2 kinds: arithmetic formulas $\pi(x) \approx \frac{x}{\ln x}$ and analytical formulas derived from the works of Riemann and Mangoldt. For example, the known formula $\operatorname{Li}(x)-\sum_{\rho} x^{\rho}-\ln 2+\int_{x}^{\infty} \frac{d t}{t\left(t^{2}-1\right) \ln t}$, where $x>1, \rho-\operatorname{non}-\operatorname{trivial}$ zeros $\zeta(s)$ functions. Since Riemann was a great specialist in the development of complex numbers and was oriented in an imaginary plane better than in real one, the learned people were at an impasse. However, the most accurate and simple formulas are rear. Since we have (Table 2) the distribution of the parameters primes \& composite numbers $\Theta$ in $\mathbb{N}$, then the law of distribution of primes $\pi(x)$ in interval $(1 \div x)$ will look like for $m=\left[\frac{x}{6}\right]$,

$$
\pi(x)=2+\sum_{\lambda=1}^{m}\left(S_{1}+S_{2}\right)
$$

Example 4. Determine the number of primes, $\pi(\mathrm{x})$ in the numerical interval, $(1 \div N)$.

$1.1 \div N=100 \rightarrow 1 \div m=\left[\frac{N}{6}\right]=16, \sum_{\lambda=1}^{\leq m} S_{1}=12, \sum_{\lambda=1}^{\leq m} S_{2}=11$, then $\pi(x)=2+12+11=25$

2. $1 \div N=558 \rightarrow 1 \div m=\left[\frac{N}{6}\right]=93, \sum_{\lambda=1}^{\leq m} S_{1}=51, \sum_{\lambda=1}^{\leq m} S_{2}=47$, then $\pi(x)=2+51+47=100, \quad$ it $\quad$ is known that in $1 \div N=558$ the $\pi(x)=100$. However in the same segment, according to the known 
function of the law of distribution of primes $\pi(x)=\frac{x}{\ln x}=\frac{558}{6.32435896} \approx 90$ it is easy to see that the result of formula (8) obtained i s closer to the truth. And no matter how large the interval of the value $x$ remains unchanged with deviation of \pm 1 .

\section{An algorithm for finding primes $p \geq 5$ in the interval $[1 \div N$ )}

Since the set of primes $P(\geq 5) \subset \Theta \subset \mathbb{N}$, it is obvious that the search for primes will go faster in the set $\Theta$ than in the set $\mathbb{N}$. The most natural way to remove composite numbers of set $\Theta$ in interval $(1 \div N)$ is by using the properties of closed elements with respect to the multiplication operationumbers Multiplying numbers of the forms $6 i-1 \& 6 i+1$ by each element of the set $\Theta$, where $i \in\left(1 \div\left[\frac{N}{6}\right]\right)$ is easy and simple to achieve the goal. First, the natural numbers are entered line by line in the file $\Theta=\operatorname{PrmNub1(id.~[N]),~but~in~the~place~the~numbers~divisible~by~} 2$ or 3 are filled with an empty symbol “-”. Then, based on the RasPrm algorithm (Fig.) the elements of the file $\Theta$ ' are deleted, those that are elementwise products of numbers $\theta_{i}=6 i-1$ are multiplied by the next (at the begining $j=i$ ) number $\theta_{j=j+1} \in \Theta$ and deleting for numbers with record numbers $i d=\theta_{i} \cdot \theta_{j}$, where $i=1,2,3, \ldots$ (see Example 5 below).

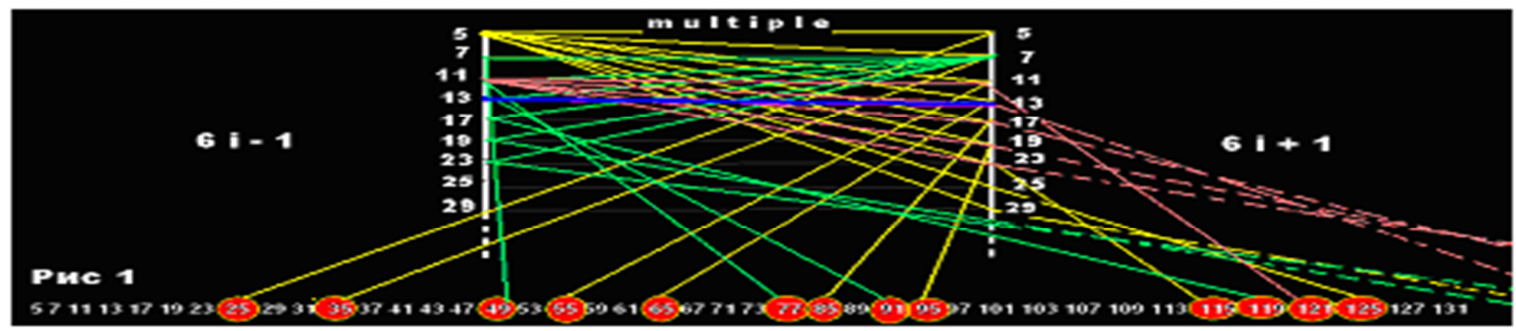

The window of the program that implements the RasPrm algorithm

Each successive new element $\theta_{i}$ erects squared to avoid repetitions of multiplication operation, and then is multiplied by the $\theta_{j=j+1}$. The removal process continues until $\theta_{i}^{2} \leq n$. If the product of the numbers $\theta_{i} \cdot \theta_{j}$ is greater than $n$, then performs the next element $\theta_{i=i+1}$ and the above procedure is repeated. Similarly, it is true for numbers of the form $\theta_{i}=6 i+1$. The method described for the elimination of composite numbers from the set $\Theta$ for numbers of the form $6 \kappa \pm 1$ is easy to use and works more efficiently and faster than such well-known algorithms as the Eratosthenes, the Sundaram and the Atkin sieves. Since in all these algorithms the domain of functioning is the set $\mathbb{N}$, the method of deleting composite numbers from the set $\Theta$ by numbers of the form $6 \kappa \pm 1$ allows to obtain the same results as the above mentioned algorithms, but with a much smaller number of multiplication operations.

Example 5. Find all prime numbers of the set $\Theta$ in the interval $1 \div N=133$.

With using numbers of the form $\theta_{i}=6 i \pm 1$, where $i \in 1 \div[133 / 6]=22$, we form the elements of the file $\Theta=\{5,7,11,13,17,19,23,25,29,31,35,37,41,43,47,49,53,55,59,61,65,67,71,73,77,79$, $83,85,89,91,95,97,101,103,107,109,113,115,119,121,125,127,131,133, \ldots\}$.

\section{Deleting composite numbers from the file $\Theta$ having the form $\theta_{i}=6 i-1$.}

Let $i=1$, then $\theta_{1}=5$. As a new $\theta_{1}$, we find its square: $\theta_{1}^{2}=25$ and if $i d=25 \leq 133$ then from the $\Theta$ by the record number $i d=25$ by direct access, this entry is raised and the field value is deleted то $[N]=25$. Then $\theta_{1}$ is multiply by the next number of the file $\Theta$ and also deleting for numbers with record numbers $i d=5 \cdot 7=35, i d=5 \cdot 11=55, \quad i d=5 \cdot 13=65, \quad i d=5 \cdot 17=85, \quad i d=5 \cdot 19=95$, $i d=5 \cdot 23=115, i d=5 \cdot 25=125$. Since $i d=5 \cdot 29>133$ we take the next step i. e. $\theta_{2}=11$ new 
element $11^{2}<133$ the record is deleted $i d=121$. When $i d=11 \cdot 13>133$, the next step $\theta_{3}=17$ new element $17^{2} \geq 133$ and terminates, the process for numbers $6 i-1$.

2. Deleting composite numbers from the file $\Theta$ having the form $\theta_{i}=6 i+1$.

Let $i=1$, then $\theta_{1}=7$ as a new element $\theta_{1}$, we find its square $i d=7^{2}=49<133$ and it is removed in the same way as in the previous examples. That is, by directly accessing the record the following record numbers are also selected $i d=7 \cdot 11=77, i d=7 \cdot 13=91, i d=7 \cdot 17=119$ and the numbers are deleted. Since id $=7 \cdot 19 \geq 133$ next step $\theta_{2}=13 \rightarrow \theta_{2}^{2}>133$.

\section{Representation of even numbers $\boldsymbol{\zeta}>\mathbf{8}$ in the form of sums of $\mathbf{2}$ elements from the $\Theta$}

Pair $\left(\lambda_{1}, \lambda_{2}\right)$ for numbers of forms $\theta_{1}=6 \lambda_{1} \pm 1 \& \theta_{2}=6 \lambda_{2} \pm 1$ are called the corresponding pair of parameters of an even number $\zeta$, if $\lambda_{1} \in \Lambda_{1}=\left[1 ;\left[\frac{v}{2}\right]\right], \lambda_{2}=\left(v-\lambda_{1}\right) \in \Lambda_{2}=\left[\left[\frac{v}{2}\right]+1 ; v\right], \zeta=\theta_{1}+\theta_{2}$.

Lemma 3. The even numbers $\zeta>8$ are comparable with $\zeta \equiv 0,2,-2(\bmod 6)$.

Evidence. From the type of even numbers, $\zeta=2 n \rightarrow 2 n / 6=n / 3$, i. e. the number $n$ has the following form $3 v+\alpha$, where $v \in \mathbb{N}$ and it is obvious that the residues $\alpha=(0,1,2)$. Suppose:

1. $\alpha=0 \rightarrow \zeta=2 n=2(3 v+0)=6 v+0 \rightarrow m=0$ that is, is divisible by 6 with the remainder 0 .

2. $\alpha=1 \rightarrow \zeta=2 n=2(3 v+1)=6 v+2 \rightarrow m=2$ that is, is divided by 6 with the remainder 2 .

3. $\alpha=2 \rightarrow \zeta=2 n=2(3 v+2)=6 v+4$ or $(m=v+1) \rightarrow 6 m-2$ i. e divisible 6 with remain -2 .

Lemma 4. Any even number $\zeta>8$ is representable by the sums of 2 elements of set $\Theta$.

Proof. Types of decomposition of even number $\zeta>8$ comparable with the $\zeta \equiv(0,2,-2)(\bmod 6)$ :

1. Let us have even numbers of the form $\zeta=6 v+0$ and let $v=\lambda_{1}+\lambda_{2}$, then, if we add and subtract 1 (one), we have an even number $\zeta=6 \lambda_{1}+6 \lambda_{2}=\left(6 \lambda_{1}+1\right)+\left(6 \lambda_{2}-1\right)=\theta_{1}^{+}+\theta_{2}{ }^{-}$.

2. Let us have even numbers of the form $\zeta=6 v+2$ and let $v=\lambda_{1}+\lambda_{2} \rightarrow$ therefore, the even number will be $\zeta=6 \lambda_{1}+6 \lambda_{2}+2=\left(6 \lambda_{1}+1\right)+\left(6 \lambda_{2}+1\right)=\theta_{1}^{+}+\theta_{2}^{+}$.

3. Let even numbers of the form be $\zeta=6 v-2$ and $v=\lambda_{1}+\lambda_{2}$ we have $\zeta=6 \lambda_{1}+6 \lambda_{2}-2=\left(6 \lambda_{1}-1\right)+\left(6 \lambda_{2}-1\right)=\theta_{1}^{-}+\theta_{2}^{-}$where the elements $\theta_{1}^{+/-}$and $\theta_{2}^{+/-}$lie in the set $\Theta$. Since the parameters of twins composite numbers $\Pi_{T w C N}$ (7) lie on the intersections of the function (5), th en the number of their parameters on the segment $[1 \div N]$ by the intersection of the sets will be less or equal than to their union the number of images of the functions themselves. Hence, on the segment $[1 \div N]$ for the parameters of the subsets: $T w, T w C N, U P C$ the following inequality is satisfied:

$$
\Pi_{T w C N}<\Pi_{U P C} \cup \Pi_{T w}
$$

consider by analogy with the remainders $m=(0,2,-2)$ decomposition of even numbers $\zeta>8$ in the form of sums $\theta_{1}+\theta_{2}$. To do this, for each type of even numbers of the form $6 v+m$ we add and subtract the numbers $6 \lambda \pm 1$.

$\alpha) \forall$ even number of the form $\zeta=6 v+0$, where $v=\frac{(\zeta-0)}{6}$, we add and remove the numbers of type $(6 \lambda-1)$, have $\zeta=6 v+[(6 \lambda-1)-(6 \lambda-1)]=(6 \lambda-1)+[6(v-\lambda)+1]$ and in the same way for numbers of form $6 \lambda+1$, then $\zeta=6 v+[(6 \lambda+1)-(6 \lambda+1)]=(6 \lambda+1)+[6(v-\lambda)-1]$. We denote the parameters of the terms $\lambda_{1}=\lambda, \lambda_{2}=v-\lambda_{1}$. Hence,

$$
\zeta=\left(6 \lambda_{1}-1\right)+\left(6 \lambda_{2}+1\right) \text { or } \zeta=\left(6 \lambda_{1}+1\right)+\left(6 \lambda_{2}-1\right)
$$


is also fulfilled the identity

$$
(6 \lambda-1)+[6(\lambda+2)+1]=(6 \lambda+1)+[6(\lambda+2)-1 .
$$

Let the even number $\zeta=96$, then $6 v=96, v=[(96-0) \backslash 6]=16$, when $\lambda_{1}=1 \& \lambda_{2}=16-1=15$ from link $(10) \rightarrow \theta_{1}^{-}=5$ and $\theta_{2}^{+}=91$ or $\theta_{1}^{+}=7$ and $\theta_{2}^{-}=89$.

$\beta) \forall$ even number of the form $\zeta=6 v+2$, where $v=(\zeta-2) / 6$ have $6 v+2+[(6 \lambda+1)-(6 \lambda+1)]=(6 \lambda+1)+(6(v-\lambda)+1)$.

We denote the parameters of the terms respectively, $\lambda_{1}=\lambda \& \lambda_{2}=v-\lambda_{1}$, which means that even number $\zeta>8$ of the form

$$
\zeta=6 v+2=(6 \lambda+1)+[6(v-\lambda)+1]=\left(6 \lambda_{1}+1\right)+\left(6 \lambda_{2}+1\right)
$$

also fulfills the identity

$$
(6 \lambda+1)+(6(\lambda+3)+1)=(6(\lambda+1)+1)+6(\lambda+2)+1) .
$$

Let $\zeta=98$, then $6 v+2=98, v=[(98-2) \backslash 6]=16$, with $\lambda_{1}=2$ and $\lambda_{2}=14$, we have from link $(12) \rightarrow \theta_{1}^{+}=13$ and $\theta_{2}^{+}=85, \zeta=\theta_{1}^{+}+\theta_{2}^{+}$.

$\gamma) \forall$ even number of the form $\zeta=6 v-2$, where the parameter $v=(\zeta+2) \backslash 6$, we have $6 v-2+[(6 \lambda-1)-(6 \lambda-1)]=(6 \lambda-1)+[6(v-\lambda)-1]$. We denote the parameters of the terms: $\lambda_{1}=\lambda \& \lambda_{2}=v-\lambda_{1}$ hence $\zeta>8$ species

$$
\zeta=6 v-2=(6 \lambda-1)+[6(v-\lambda)-1]=\left(6 \lambda_{1}-1\right)+\left(6 \lambda_{2}-1\right)
$$

also fulfills the identity

$$
(6 \lambda-1)+[6(\lambda+3)-1]=[6(\lambda+1)-1]+[6(\lambda+2)-1] .
$$

Suppose that $\zeta=100 \rightarrow 6 v-2=100, v=[(100+2) \backslash 6]=17$ for $\lambda_{1}=3$, we have $\lambda_{2}=14$ from link (14) $\rightarrow \theta_{1}^{-}=17$ and $\zeta=\theta_{1}^{-}+\theta_{2}^{-}, \theta_{2}^{-}=83$.

From the properties $\alpha, \beta, \gamma$ it follows that $\forall$ even number $\zeta<8$ can be represented by sums $\left(6 \lambda_{1} \pm 1\right)+\left(6 \lambda_{2} \pm 1\right)$, where $\lambda_{1} \in \Lambda_{1}, \lambda_{2}=\left(v-\lambda_{1}\right) \in \Lambda_{2}$ and also if $m$ is the remainder of even numbers divided by 6 , then the parameter of the even number $\zeta<8$ is found by the formula

$$
v=(\zeta-m) \backslash 6 .
$$

\section{The binary (strong) Goldbach - Euler problem}

The decomposition of even numbers $\zeta<8$ into the sum of 2 primes is verified directly, so consider the solution of binary Goldbach problem for $\zeta>8$. We first introduce the definition and prove a number of lemmas.For example to find the corresponding parameters of the even numbers $\zeta=30$. Since, $30 \equiv 0(\bmod 6)$, then the parameter of even numbers $v=\frac{30-0}{6}=5$. Hence, $\lambda_{1} \in \Lambda_{1}=[1,2], \lambda_{2} \in \Lambda_{2}=[3,4,5]$.

1. Let $\lambda_{1}=1, \lambda_{2}=v-\lambda_{1}=5-1=4, \rightarrow \theta_{1}=6 \cdot 1-1=5, \theta_{2}=6 \cdot 4+1=25$ or $\theta_{1}=6 \cdot 1+1=7$, $\theta_{2}=6 \cdot 4-1=23$. Since, $5+25=7+23=30$ corresponding parameters $\left(\lambda_{1}, \lambda_{2}\right)=(1 ; 4)$. 
2. Let $\lambda_{1}=2$, then $\lambda_{2}=v-\lambda_{1}=5-2=3, \theta_{1}=6 \cdot 2-1=11, \theta_{2}=6 \cdot 3+1=19$ or $\theta_{1}=6 \cdot 2+1=13$ and $\theta_{2}=6 \cdot 3-1=17$. Since $11+19=13+17=30$, then the corresponding pair paramets $\left(\lambda_{1}, \lambda_{2}\right)=(2 ; 3)$. Thus, even $\zeta=30$ has 2 corresponding parameters $\left(\lambda_{1}, \lambda_{2}\right)=\{(1 ; 4),(2 ; 3)\}$.

Lemma 5. $\forall$ even numbers $\zeta>8$ in the segments $\Lambda_{1}, \Lambda_{2}$ there always exists parameters $\lambda_{1} \in \Lambda_{1}, \lambda_{2}=\left(v-\lambda_{1}\right) \in \Lambda_{2}$, which belong to the union of the sets $\Pi_{T w} \cup \Pi_{U P C}$ and are corresponding pair parameters of even numbers.

Evidence. The parameters of twins of composite numbers are increasing in segment $[1 \div v]$, since the intersection of the functions (5) is increasing, then in the segments $\Lambda_{1}$ and $\Lambda_{2}$ naturally, they will be have different elements $\Pi_{T w C N}$. Since, the natural numbers in the segment $\Lambda_{1}$ begin with the parameters of twins of primes $\Pi_{T w}=\{1,2,3,5,7,10,12,17,18,23,25, \ldots\}$ (see Table 6 below or Table 5 in [6]) then all elements of the segment $\Lambda_{1}$, obviously, can not be parameters of twins of composite numbers. Suppose that all elements of the segment $\Lambda_{2}$ are parameters of twins of composite numbers, then by (9) we have a contradiction, since the total number of elements of $\Pi_{T w C N}$ on the segm. $[1 \div v]$, can't be greater than the number of elements $\Pi_{U P C} \cup \Pi_{T w}$. Hence, the assumption is false, that is, in the segments $\Lambda_{1}$ and $\Lambda_{2}$, the number of parameters $\left|\Pi_{T w C N}\right|<\left|\Pi_{U P C} \cup \Pi_{T w}\right|$. If in segment $\Lambda_{1}$ the number of parameters of twins of composite numbers is equal to $k_{1}$ and in segment $\Lambda_{2}$ is $k_{2}<\left[\frac{v}{2}\right]$, then the number of parameters $\left|\Pi_{T w} \cup \Pi_{U P C}\right|$ in $\Lambda_{1}$ is $\delta_{1}=\left(\left[\frac{v}{2}\right]-k_{1}\right)$ and in $\Lambda_{2}: \delta_{2}=\left(\left[\frac{v}{2}\right]-k_{2}\right)$. Let $k_{1}<k_{2}$, then it is obvious that $\delta_{1}>\delta_{2}$. Let the parameters $\left(\lambda_{1}^{1}, \lambda_{1}^{2}, \ldots, \lambda_{1}^{k_{1}}\right) \in \Lambda_{1} \in \Pi_{T w C N}$ and assume that (to strengthen the assertion of Lemma 5) the corresponding pair parameters $\left(\lambda_{2}^{1}, \lambda_{2}^{2}, \ldots, \lambda_{2}^{k_{1}}\right) \in \Lambda_{2}$ are elements of $\Pi_{T w} \cup \Pi_{U P C}$. Similar reasoning on the parameters is found in segment $\Lambda_{2}$. Then, the numbers $\delta_{1}$ or $\delta_{2}$ for parameters $\left|\Pi_{T w} \cup \Pi_{U P C}\right|$ in the segment $\Lambda_{1}$ or $\Lambda_{2}$ will be $\left(\left[\frac{v}{2}\right]-k_{1}\right)-k_{2}=\left[\frac{v}{2}\right]-\left(k_{1}+k_{2}\right)$, where $\left(k_{1}+k_{2}\right)$ shows the number of corresponding pair parameters, one of which the parameter is of twins of composite numbers on the segment $[1 \div v]$. Because the in the segments $\Lambda_{1}$ and $\Lambda_{2}$ the number of parameters of twins of composite numbers of is less than the number of elements $\left|\Pi_{T w} \cup \Pi_{U P C}\right|$, then naturally in $\Lambda_{1}$ and $\Lambda_{2}$ there is always a pair of numbers $\lambda_{1} \in \Lambda_{1}, \lambda_{2} \in \Lambda_{2}$ such $\left(\lambda_{1}, \lambda_{2}\right) \in \Pi_{T w} \cup \Pi_{U P C}$ and are the corresponding pairs of parameters of an even number $\zeta>8$.

Lemma 6. In segm. $[1 \div v]$ the number of elements $\left|\boldsymbol{\Pi}_{T w} \cup \boldsymbol{F N ^ { - }}\right| \geq\left|\boldsymbol{F N ^ { + }}\right|,\left|\boldsymbol{\Pi}_{T_{w}} \cup \boldsymbol{F N ^ { + }}\right| \geq\left|\boldsymbol{F} \boldsymbol{N}^{-}\right|$

Evidence. From the contents of the procedure for constructing the (see Table 1) it is obvious that the ele ments of the sets $F N^{+}=f_{11}(x, y) \cup f_{12}(x, y)$ and $F N^{-}=f_{21}(x, y) \cup f_{22}(x, y)$ in each row give rise to 2 elements, that is, in the segment $[1 \div v]$ the number of elements $\left|F N^{+}\right| \sim\left|F N^{-}\right|$. Hence, the following inequalities are true $\left|\Pi_{T w} \cup F N^{-}\right| \geq\left|F N^{+}\right|,\left|\Pi_{T_{w}} \cup F N^{+}\right| \geq\left|F N^{-}\right|$.

\section{Theorem 3. Any even number $\zeta>8$ is decomposable into a sum of two primes.}

Evidence. Since, any even numbers of the form $\zeta=6 v+m$ and $\zeta>8$ are considered and following from (16) that the values of the parameters of the even numbers $v=\left[\frac{\zeta-m}{6}\right] \geq 1$, for the 
remainders $m=(0,2,-2)$. Then, by analogy with the remainders $m$, the even numbers $\zeta>8$ are represented by the sums $\theta_{1}+\theta_{2}$, where $\theta_{1}=6 \lambda_{1} \pm 1$ and $\theta_{2}=6 \lambda_{2} \pm 1,\left(\lambda_{2}=v-\lambda_{1}\right)$ by the types of expansions of even numbers $\zeta>8$ (Lemma 4) or by properties $\alpha, \beta, \gamma$ we have the following structures of the corresponding forms of the decomposition of even numbers $\zeta>8$ :

$$
\left\{\begin{array}{ll}
\text { a). } \zeta=6 v+0: & \theta_{1}=\left\{6 \lambda_{1} \pm 1 \mid \lambda_{1} \in \Lambda_{1}\right\} \text { and } \theta_{2}=\left\{6 \lambda_{2} \pm 1 \mid \lambda_{2} \in \Lambda_{2}\right\} \\
\text { b). } \zeta=6 v+2: & \theta_{1}=\left\{6 \lambda_{1}+1 \mid \lambda_{1} \in \Lambda_{1}\right\} \text { and } \theta_{2}=\left\{6 \lambda_{2}+1 \mid \lambda_{2} \in \Lambda_{2}\right\} \\
\text { c). } \zeta=6 v-2: & \theta_{1}=\left\{6 \lambda_{1}-1 \mid \lambda_{1} \in \Lambda_{1}\right\} \text { and } \theta_{2}=\left\{6 \lambda_{2}-1 \mid \lambda_{2} \in \Lambda_{2}\right\}
\end{array}\right\} .
$$

We investigate the elements of the segment $[1 \div v]$ for belonging to the sets $\Pi_{T w}, \Pi_{T w C N}, \Pi_{U P C}$ and find out for which values of the parameters $\lambda_{1} \in \Lambda_{1}$ and $\lambda_{2} \in \Lambda_{2}$ from the listed sets the numbers $\theta_{1}=6 \lambda_{1} \pm 1$ and are primes or for which composite numbers. For elements of the set $\Pi_{T w C N}$ they are not considered, since $\theta_{1}, \theta_{2}$ is composite numbers. However, by Lemma 5 in segment $[1 \div v]$ there are always $\lambda_{1} \in \Lambda_{1}$ and $\lambda_{2} \in \Lambda_{2}$ such that $\lambda_{1}, \lambda_{2} \in\left(\Pi_{T w} \cup \Pi_{U P C}\right)$, then $\left(\lambda_{1}, \lambda_{2}\right) \in \Pi_{T w} \cup\left(F N^{+} \Delta \boldsymbol{F N}^{-}\right)$. Hence it remains to verify the elements $\lambda_{1} \in \Lambda_{1}, \lambda_{2} \in \Lambda_{2}$, on belonging to the sets $\Pi_{T w}$ and $F N^{+} \Delta \boldsymbol{F N}^{-}$ with the removed parameters of set $\Pi_{T w C N}$ in the segment $[1 \div v]$, we have:

A. Let $\lambda_{1} \in \Pi_{T w}, \lambda_{2} \in \Pi_{T w}$, then the terms $\theta_{1}, \theta_{2}$ of the even numbers $\zeta>8$ are primes, as the constituent elements of twins of primes $\theta_{1}=\left(\begin{array}{c}p_{1}=6 \lambda_{1}-1, \\ p_{1}=6 \lambda_{1}+1\end{array}\right)$ and $\theta_{2}=\left(\begin{array}{c}p_{2}=6 \lambda_{2}-1, \\ p_{2}=6 \lambda_{2}+1\end{array}\right)$. Hence, according to the corresponding forms in (17) we have $\zeta=p_{1}+p_{2}$ and infinity of twins of primes I will soon publish.

B. $\operatorname{Let}\left(\lambda_{1}\right.$ or $\left.\lambda_{2}\right) \in \Pi_{T w} \&\left(\lambda_{2}\right.$ or $\lambda_{1} \in\left(F N^{+} \Delta \boldsymbol{F} N^{-}\right)$so the term $\theta_{1}$ is prime numbers as constituent elements of twins of primes $\theta_{1}=\left(p_{1}=6 \lambda_{1}-1, p_{1}^{\prime}=6 \lambda_{1}+1\right)$. And the 2 nd term $\theta_{2}=6 \lambda_{2} \pm 1$ by Lemma 2 in one of the variants of the forms: $\theta_{2}=\left(p_{2}=6 \lambda_{2}-1\right.$ or $\left.p_{2}^{\prime}=6 \lambda_{2}+1\right)$ is a prime numbers. Then adding to the prime numbers $\theta_{2}=p_{2}$ obtained a prime numbers $\theta_{1}=p_{1}$ (by analogy with the corresponding from (17), we get $\zeta=p_{1}+p_{2}$ and infinity of twins of primes will soon be published.

C. Let $\left(\lambda_{1}, \lambda_{2} \in\left(F N^{+} \Delta \boldsymbol{F} N^{-}\right)\right.$, then in order that the term $\theta_{1}$ of an even numbers $\zeta>8$, depending on the corresponding form defined in (17) and by Lemma 2 be a prime numbers, it is sufficient to establish whether the parameter $\lambda_{1}$, belongs to one of the sets $\left(F N^{-}\right.$or $\left.F N^{+}\right)$. For the term $\theta_{2}$ the parameter of which $\lambda_{2}=v-\lambda_{1}$ on the segment $[1 \div v]$ can obviously correspond to the parameters $\Pi_{T w}$ or again the elements from the set $\left(F N^{+} \Delta \boldsymbol{F} \boldsymbol{N}^{-}\right)$. If $\lambda_{2} \in \Pi_{T w}$, then there are no problems, otherwise the following parameter $\lambda_{1}$ is determined by the identities (11), (13), (15), respectively, by type of an even numbers $6 \mathrm{v}+m$, which is obviously an element of either $\Pi_{T w}$ or $\left(F N{ }^{+} \Delta F N^{-}\right)$. But since by Lemma 6 the number of parameters in segments $[1 \div v]\left|\Pi_{T w} \cup F N^{-}\right| \geq\left|F N^{+}\right|$and $\left|\Pi_{T w} \cup F N^{+}\right| \geq\left|F N^{-}\right|$, then one of the items (A, B) is repeated in $\Lambda_{1} \& \Lambda_{2}$. Thus, the decomposition of an even number $\zeta>8$ by the sum of 2 numbers $\theta_{1}+\theta_{2}$, where the numbers of form $\theta_{1}=6 \lambda_{1} \pm 1, \theta_{2}=6 \lambda_{2} \pm 1$, whose parameters $\left(\lambda_{1}\right.$ and $\left.\lambda_{2}\right) \in\left(\Pi_{T w} \cup \Pi_{U P C}\right)$ in any of the listed variants $(\mathrm{A}, \mathrm{B}, \mathrm{C})$ always lead to the forms $6 \lambda_{1} \pm 1$ and $6 \lambda_{2} \pm 1$ of primes and since the sum of the numbers $\theta_{1}+\theta_{2}$ is even numbers, either $6\left(\lambda_{1}+\lambda_{2}\right)$ or $6\left(\lambda_{1}+\lambda_{2}\right) \pm 2$, then the Goldbach Euler binary problem $\zeta=p_{1}+p_{2}$ is solved in any case positively.

Example 6. Let an even number $\zeta=360$. Let us calculate the remainder $m$ from dividing an even numbers $\zeta$ by 6 . By Lemma 3 we find the type of an even number $6 v+0$, and by (17), respectively, we have the string $(17, a)$. We establish the forms of the terms in the expansion of the even numbers $\zeta>8$, 
we have numbers of the form $\theta_{1}=6 \lambda_{1} \pm 1, \theta_{2}=6 \lambda_{2} \pm 1$, we calculate the even numbers' parameter by the $v=\frac{\zeta-m}{6}$, where the remainder $m=0$ whence $v=60$. We write out the elements of the sets $\Pi_{T w C N}, \Pi_{T w}, \Pi_{U P C}$ from the corresponding tables on the segment $[1 \div 60] \Pi_{T w C N}^{*}=\{20,24,31,34,36$, $41,48,50,54,57\}$ (see Table 5 below or Table 6 [3]).

$\Pi_{T w}^{\vee}=N F N=\{1,2,3,5,7,10,12,17,18,23,25,30,32,33,38,40,45,47,52,58\}$ (see Table 6 below or Table 5 [3]).

$F N^{-}=\{6,11,13,16, * 20,21, * 24,26,27, * 31, * 34,35, * 36,37, * 41,46, * 48, * 50,51, * 54,55$, $56, * 57\}$ (Table 1).

$F N^{+}=\{4,8,9,14,15,19, * 20,22, * 24,28,29, * 31, * 34, * 36,39, * 41,42,43,44, * 48,49, * 50$, $53, * 54,55, * 57,59,60\}$. Symbols in numbers according to belonging to sets $\Pi_{T w C N}^{*}, \Pi_{T w}^{\vee}, F N^{-}, F N^{+}$. We decompose the elements of the corresponding pairs of parameters lieing in $\lambda_{1} \in\left[1 \div\left[\frac{\nu}{2}\right]=30\right]$ and $\lambda_{2} \in[30 \div 60]$ taking into account their belonging to $\Pi_{T w C N}, \Pi_{T w}, \Pi_{U P C}$ parameter sets, according to $\boldsymbol{\Lambda}_{1}=\left\{\underline{1}, \underline{2}, \underline{3},{ }^{+} 4, \underline{5},-6, \underline{7},{ }^{+} 8,{ }^{+} 9, \underline{10},{ }^{-} 11, \underline{12},{ }^{-13},{ }^{+} 14,{ }^{+} 15,{ }^{-} 16, \underline{17}, \underline{18},{ }^{+} 19, * 20,{ }^{-} 21,{ }^{+} 22, \underline{23}, * 24, \underline{25}\right.$, $\left.{ }^{-} 26,{ }^{-} 27,{ }^{+} 28,{ }^{+} 29, \underline{30}\right\} . \Lambda_{2}=\left\{* 31, \underline{32}, \underline{33}, * 34,{ }^{-} 35, * 36,{ }^{-} 37, \underline{38},{ }^{+} 39, \underline{40}, * 41,{ }^{+} 42,{ }^{+} 43,{ }^{+} 44, \underline{45},{ }^{-} 46, \underline{47}\right.$, $\left.* 48,{ }^{+} 49, * 50,{ }^{-} 51,52,{ }^{+} 53, * 54,-55,-56, * 57,58,{ }^{+} 59,{ }^{+} 60\right\}$.

The numbers of corresponding pairs of parameters $\left(\lambda_{1}, \lambda_{2}\right)$ lie in segment $[1 \div v]$ and, naturally, for the parameters of the numbers $\Pi_{T w C N}$, the corresponding summands are composite numbers, but we are not interested in such. Then, it will be necessary to choose from $\Lambda_{1}$, and $\Lambda_{2}$ those parameters that belong to the union of the sets $\Pi_{T w} \cup\left(F N^{+} \Delta \boldsymbol{F N}-\right)$. For example, suppose the parameter values are:

$\begin{array}{lll}\lambda_{1}=2 \in \Pi_{T w} & 6 \lambda_{1}-1=11 \in P N^{-}, & 6 \lambda_{1}+1=13 \in P N^{+}, \\ \lambda_{2}=58 \in \Pi_{T w} & 6 \lambda_{2}+1=349 \in P N^{+} & 6 \lambda_{2}+1=347 \in P N^{-} \\ \lambda_{1}=25 \in \Pi_{T w} & 6 \lambda_{1}-1=149 \in P N^{-}, & 6 \lambda_{1}+1=151 \in P N^{+}, \\ \lambda_{2}=35 \in F N^{-} & 6 \lambda_{2}+1=211 \in P N^{+} & 6 \lambda_{2}-1=209 \in C N^{-} \\ \lambda_{1}=5 \in \Pi_{T w} & 6 \lambda_{1}-1=29 \in P N^{-} & 6 \lambda_{1}+1=31 \in P N^{+}, \\ \lambda_{2}=55 \in F N^{+} & 6 \lambda_{2}+1=331 \in C N^{+} & 6 \lambda_{2}-1=329 \in P N^{-} \\ \lambda_{1}=46 \in F N, & 6 \lambda_{1}-1=275 \in C N^{-} & 6 \lambda_{1}+1=277 \in P N^{+}, \\ \lambda_{2}=14 \in F N^{+} & 6 \lambda_{2}+1=85 \in C N^{+} & 6 \lambda_{2}-1=83 \in P N^{-} \\ \lambda_{1}=39 \in F N^{+}, & 6 \lambda_{1}-1=233 \in P N^{-}, & 6 \lambda_{1}+1=235 \in C N^{+}, \\ \lambda_{2}=21 \in F N^{-} & 6 \lambda_{2}+1=127 \in P N^{+} & 6 \lambda_{2}-1=125 \in C N^{-}\end{array}$

Since the parameters $\lambda_{1}=\{1,2,5,23,25, \ldots\} \in \Pi_{T w}$ on $[1 \div 30]$, then the numbers of the form $\theta_{1}=6 \lambda_{1} \pm 1$ are primes as of twins of primes, i. e. $(5-7,11-13,29-31,137-139,149-151)$ and the corresponding parameters $\lambda_{2} \in[30 \div 60]$ in one of the variants of the forms $\theta_{2}=6 \lambda_{2}-1$ or $\theta_{2}=6 \lambda_{2}+1$ are prime numbers, by Lemma 2 . Thus, even number 360 is decomposable into sums of primes $11+349,13+347,149+211,29+331,277+83,233+127$.

Example 7. Let an even number $\zeta=362$. We compute the remainder $m$ from dividing an even number $\zeta$ by 6 , and by Lemma 3 we find the type of an even number $6 v+2 \rightarrow m=2$, according to (17), respectively, we have the string $(17, b)$. We fix the forms of the terms in the expansion of an even number $\zeta>8$, we have numbers of the form $\theta_{1}=6 \lambda_{1}+1 ; \theta_{2}=6 \lambda_{2}+1$. We calculate the even numbers' parameter by the formula (16), where $v=60$. We write out the elements of the subsets $\Pi_{T w C N}, \Pi_{T w}$, $\Pi_{U P C}$ from the corresponding tables on the segment $[1 \div 60]$. It is obvious that the values of the parameters of the sets $\Pi_{T w}, \Pi_{T w C N}, F N^{+}, F N^{-}$and $\Lambda_{1}, \Lambda_{2}$ remain unchanged, as in Example 6, but since the numbers here are of the same type, in order that $\theta_{1}, \theta_{2}$ are primes, it is necessary that the parameters $\lambda_{1}, \lambda_{2}$ were taken from the sets $\Pi_{T w}$ or $F N^{-\mid} \Pi_{T w C N}$ :

$$
\begin{array}{clll}
\lambda_{1}=23 \in \prod_{T_{w}} & \lambda_{2}=37 \in F N^{-} & 6 \lambda_{1}+1=139 \in P N^{+} & 6 \lambda_{2}+1=223 \in P N^{+} \\
\lambda_{1}=27 \in F N^{-} & \lambda_{2}=33 \in \prod_{T_{w}:}: & 6 \lambda_{1}+1=163 \in P N^{+} & 6 \lambda_{2}+1=199 \in P N^{+} \\
\lambda_{1}=6 \in F N^{-} & \lambda_{2}=14 \in F N^{+} & 6 \lambda_{1}+1=277 \in P N^{+} & 6 \lambda_{2}+1=85 \in C N^{+} \\
\lambda_{1}=47 \in \prod_{T_{w}} & \lambda_{2}=13 \in F N^{-} & 6 \lambda_{1}+1=283 \in P N^{+} & 6 \lambda_{2}+1=79 \in P N^{+}
\end{array}
$$


Thus, the number 362 is decomposable into sums of 2 primes: $139+223,163+199,283+79$.

Table 5

\section{Parameters of twins of composite pairs $\left(\Pi_{T w C N}\right)$ in set $\Theta \quad 1 \div 10000$}

020, 024, 031, 034, 036, 041, 048, 050, 054, 057, 069, 071,079, 086, 088, 089, 092, 097, 104, 106, 111, 116, 119, 130, 132, 134, 136, $139,141,145,149,150,154,160,167,171,174,176,179,180,189,190,191,193,196,201,207,209,211,212,219,222,223,224$, $225,226,231,232,234,236,244,246,251,253,256,265,272,274,275,279,280,281,284,286,288,294,295,299,301,303,306$, $307,309,314,316,320,321,323,324,326,327,328,337,339,341,343,345,349,351,353,354,358,361,362,364,365,366,371$, $372,376,377,384,386,387,388,394,401,405,409,414,415,416,418,419,421,427,428,429,431,433,434,438,440,442,454$, 456, 460, 462, 464, 468, 469, 471, 478, 479, 482, 487, 489, 491, 496, 497, 498, 499, 501, 505, 509, 512, 516, 517, 519, 521, 522, 524, $525,526,529,533,539,540,541,544,546,547,548,549,556,559,561,563,564,566,567,570,571,573,574,579,580,581,584$, $587,592,594,596,598,600,608,609,611,614,619,624,625,626,629,631,635,636,638,640,643,645,649,650,656,659,660$, $662,663,664,666,672,673,674,677,678,681,684,686,687,691,694,695,697,698,699,701,704,706,708,711,713,717,718$ $719,720,722,724,728,730,731,734,736,738,739,745,746,748,750,755,756,757,759,762,763,768,769,771,772,778,781$, $783,785,786,790,791,794,795,796,801,804,806,807,808,809,811,814,816,819,821,827,830,836,838,839,841,844,845$, $849,854,855,856,857,860,864,867,869,870,874,875,876,878,881,882,886,888,890,893,894,895,896,904.909,910,911$, $915,916,919,923,924,925,931,933,934,935,936,938,939,944,946,951,954,955,959,960,961,962,966,972,979,981,982$, 985, 986, 989, 991, 993, 994, 995, 996, 999, 1000, 1003, 1004, 1010, 1014, 1016, 1018, 1021, 1023, 1026, 1028,1030, 1031, 1032, 1039, $1040,1042,1047,1049,1051,1058,1064,1067,1068,1069,1072,1073,1074,1076,1077,1081,1083,1084,1085,1086,1089,1090$, $1093,1098,1099,1102,1104,1105,1107,1108,1111,1114,1116,1119,1121,1124,1125,1126,1128,1129,1131,1133,1135,1136$, $1141,1142,1146,1148,1149,1154,1155,1156,1157,1159,1168,1172,1175,1177,1179,1181,1182,1183,1186,1189,1190,1191$, $1194,1195,1197,1200,1204,1210,1211,1212,1213,1215,1217,1219,1221,1223,1224,1226,1227,1229,1230,1231,1233,1234$ $1237,1238,1240,1241,1244,1245,1249,1252,1259,1261,1266,1269,1271,1272,1276,1277,5192,1285,1289,1291,1294,1295$, $1296,1297,1300,1301,1302,1306,1308,1310,1315,1316,1319,1324,1326,1328,1329,1330,1331,1333,1334,1337,1338,1339$, $1341,1344,1346,1351,1355,1356,1357,1359,1364,1366,1367,1369,1371,1375,1376,1380,1384,1387,1389,1390,1391,1393$, $1397,1399,1400,1401,1402,1406,1409,1412,1413,1414,1415,1416,1418,1422,1425,1426,1428,1431,1432,1434,1436,1439$, $1442,1443,1454,1461,1462,1465,1466,1469,1471,1474,1476,1470,1480,1483,1484,1485,1486,1491,1493,1496,1497,1498$, $1499,1503,1504,1506,1509,1512,1513,1514,1516,1519,1520,1524,1528,1532,1536,1539,1541,1542,1544,1545,1548,1550$, $1551,1555,1559,1560,1561,1564,1568,1571,1574,1575,1576,1581,1584,1586,1588,1593,1594,1595,1596,1597,1599,1601$, $1606,1609,1611,1612,1614,1617,1618,1619,1621,1626,1627,1629,1633,1637,1641,1644,1646,1649,1652,1653,1656,1659$, $1660,1663,1664,1665,1666,1667,1669,1670,1671,1672,1674,1675,1676,1679,1681,1686,1687,1688,1691,1698,1700,1701$, $1703,1705,1706,1713,1714,1716,1718,1721,1725,1727,1729,1730,1731,1734,1735,1736,1737$,

Table 6

\section{Parameters of twins prime pairs $\left(\Pi_{T w}\right) \quad 1 \div 50000$}

001, 002, 003, 005, 007, 010, 012, 017, 018, 023, 025, 030, 032, 033, 038, 040, 045, 047, 052, 058, 070, 072, 077, 087, 095, 100, 103, 107, 110, $135,137,138,143,147,170,172,175,177,182,192,205,213,215,217,220,238,242,247,248,268,270,278,283,287,298,312,313,322$, $325,333,338,347,348,352,355,357,373,378,385,390,397,425,432,443,448,452,455,465,467,495,500,520,528,542,543,550,555$, $560,562,565,577,578,588,590,593,597,612,628,637,642,653,655,667,670,675,682,688,693,703,705,707,710,712,723,737,747$ $753,758,773,775,787,798,800,822,828,835,837,850,872,880,903,907,913,917,920,940,942,943,957,975,978,980$,

$1015,1022,1033,1045,1050,1060,1075,1092,1095,1110,1115,1117,1127,1130,1132,1138,1145,1158,1160,1188,1202,1218,1222$, $1225,1243,1248,1258,1260,1265,1293,1313,1325,1335,1348,1370,1372,1382,1398,1405,1423,1433,1438,1470,1473,1477,1495$, $1500,1502,1507,1540,1547,1557,1570,1572,1573,1577,1605,1613,1620,1628,1643,1655,1668,1673,1678,1682,1690,1712,1717$, $1722,1738,1743,1750,1755,1785,1810,1815,1823,1843,1845,1853,1860,1862,1892,1915,1925,1950,1953,1963,1972,1990,1995$, $2007,2012,2018,2027,2040,2042,2063,2090,2102,2137,2153,2167,2168,2203,2223,2233,2280,2282,2285,2287,2293,2305,2313$, $2317,2322,2333,2335,2347,2375,2387,2398,2408,2425,2427,2432,2438,2478,2523,2545,2548,2555,2560,2597,2607,2608,2622$, $2623,2648,2662,2677,2678,2690,2698,2705,2727,2742,2772,2775,2782,2805,2817,2830,2838,2865,2882,2898,2903,2915,2930$, 2933, 2943, 2947, 2958, 2965, 2973, 2985, 2987, 2993, 2998, 3007, 3008, 3010, 3020, 3022, 3042, 3048, 3052, 3087, 3090, 3152, 3153, 3180, $3190,3197,3202,3230,3237,3238,3245,325,3283,3292,3307,3315,3332,3337,3358,3372,3393,3407,3413,3418,3425,3440,3453$, $3458,3462,3468,3483,3497,3502,3503,3510,3532,3553,3563,3582,3587,3593,3598,3600,3602,3608,3623,3640,3673,3682,3685$, 3693, 3712, 3713, 3728, 3747, 3757, 3762, 3770, 3773, 3783, 3790, 3810, 3827, 3838, 3840, 3843, 3867, 3882, 3895, 3923, 3927, 3938, 3945, 3948, 3957, 3972, 3985, 4018, 4030, 4062, 4070, 4153, 4163, 4172, 4195, 4217, 4218, 4235, 4245, 4263, 4267, 4300, 4308, 4322, 4333, 4352, $4375,4377,4447,4450,4452,4455,4477,4480,4482,4492,4510,4518,4540,4547,4568,4580,4588,4590,4597,4615,4623,4625,4632$, 4653, 4657, 4683, 4685, 4697, 4713, 4718, 4725, 4735, 4758, 762, 4770, 4777, 4792, 4837, 4855, 4868, 4898, 4900, 4928, 4945, 4960, 4980, $5002,5015,5023,5045,5065,5078,5082,5093,5140,5142,5145,5180,5187,5192,5197,5208,5220,5232,5252,5257,5287,5288,5295$, $5308,5338,5343,5353,5357,5365,5383,5387,5395,5402,5407,5422,5427,5435,5453,5467,5472,5485,5490,5495,5512,5525,5530$ $5548,5555,5558,5598,5600,5603,5625,5628,5635,5638,5672,5688,5693,5702,5710,5717,5728,5745,5750,5752,5765,5775,5793$, $580,5808,5827,5842,5847,5880,5908,5918,5922,5932,5955,5967,5973,5983,6002,6018,6057,6078,6088,6130,6132,6150,6155$, $6170,6200,6218,6223,6227,6258,6262,6265,6282,6297,6302,6332,6373,6388,6408,6410,6428,6435,6442,6445,6452,6458,6487$, $6507,6527,6538,6540,6557,6562,6585,6638,6640,6673,6688,6692,6738,6755,6773,6783,6808,6857,6863,6867,6872,6898,6902$, $6920,6935,6960,6975,6993,6997,7003,7012,7030,7037,7047,7068,7077,7095,7107,7117,7140,7150,7175,7220,7233,7257,7263$, $7268,7275,7297,7298,7315,7327,7338,7348,7355,7367,7378,7380,7397,7422,7437,7450,7462,7520,7523,7530,7553,7557,7598$, $7637,7675,7682,7697,7712,7718,7725,7740,7765,7780,7795,7803,7805,7843,7858,7892,7898,7903,7943,7950,7952,7957,7963$, $7968,8020,8052,8068,8080,8090,8108,8113,8122,8130,8137,8143,8145,8165,8172,8187,8195,8200,8213,8222,8228,8232,8235$, $8255,8258,8278,8290,8298,8320,8323,8332$,

Example 8. Let an even number $\zeta=364$. We compute the remainder $m$ from dividing an even number $\zeta$ by 6 and by Lemma 3, we find the type of even numbers $6 v-2 \rightarrow m=-2$ and by (17), respectively, we have the string $(17, \mathrm{c})$. We fix the forms of the terms in the expansion of an even number $\zeta=8$, we have numbers of the form $\theta_{1}=6 \lambda_{1}-1$ and $\theta_{2}=6 \lambda_{2}-1$. We calculate the even 
numbers' parameter by the formula (16), whence $v=61$. Then the values of the parameters of the sets $\Pi_{T w}, \Pi_{T w C N}, F N^{+}, F N^{-}$and $\Lambda_{1}, \Lambda_{2}$ remain almost unchanged, as in Example 6, only the parameters 61 are added, that is, $[1 \div 61]$. Since here are again the numbers of one kind, then, in order for numbers of the form terms $\theta_{1}, \theta_{2}$ to be primes obviously by Lemma 3 the parameters $\lambda_{1}, \lambda_{2}$ must belong to one of the sets: $\Pi_{T w}$ or $F N^{-} \mid \Pi_{T w C N}$ :

$$
\begin{array}{llll}
\lambda_{2}=2 \in \Pi_{T W} & \lambda_{2}-1=59 \in F N^{+} & 6 \lambda_{2}-1=11 \in P N^{-} & 6 \lambda_{2}-1=353 \in P N^{-} \\
\lambda_{2}=19 \in \Pi_{T W} & \lambda_{1}-1=42 \in F N^{+} & 6 \lambda_{1}-1=113 \in P N & 6 \lambda_{1}-1=25 \in P N \\
\lambda_{2}=46 \in F N^{-} & \lambda_{2}-1=15 \in F N^{+} & 6 \lambda_{1}-1=275 \in C N^{-} & 6 \lambda_{2}-1=89 \in P N^{-}
\end{array}
$$

Thus, the number 362 is decomposable into sums of 2 primes: $11+353,113+251$. In Examples 7 and 8 we have the same type of terms $\theta_{1}$, and $\theta_{2}$. And since inequality (17) is always feasible on $\Lambda_{1}$ and $\Lambda_{2}$, the elements of the sets $\Pi_{T w}, F N^{+}, F N^{-}$are always present in $\lambda_{1}, \lambda_{2}$. Therefore, the validity of the Goldbach - Euler binary problem as a whole remains valid for all even numbers $\zeta>8, \Lambda_{1}=\left[1 \div\left[\frac{v}{2}\right]\right]$ and $\Lambda_{2}=\left[\left[\frac{v}{2}\right] \div v\right]$. It is proved that any even numbers $\zeta>8$ decomposes into a sum of 2 primes, \& since the expansion of even numbers $\zeta \leq 8$ is verified directly, the binary Goldbach - Euler problem for even numbers $\zeta>2$ is proved.

\section{Conclusion}

A comprehensive study of the problem of finding and distributing primes and composite numbers, twins of primes, twins of composite numbers, including a theoretical study by means of computer software made it possible to obtain great results, to solve the secrets of the structures of subsets of the set of natural numbers and to give the definitions, to obtain a new algorithm for finding and distributing primes $P(\geq 5)$, to fulfil the calculation of the exact number of primes and to obtain the method of distribution primes $p \geq 5, p_{n}=6 \cdot i d+(-1)^{\varphi(n)}$ by their ordinal numbers $n$ and vice versa in the set of primes. The method of their distribution law $\pi(x)=2+\sum_{\lambda=1}^{m}\left(S_{1}+S_{2}\right), m=\left[\frac{N}{6}\right]$ in interval $[1 \div N)$ has been found.

Any even number $\zeta>8$ has the form $\zeta=6 v+m$, where $v=(\zeta-m) / 6 v=(\zeta-m) / 6$ is an even parameter.

Any even number $\zeta>8$ can be represented by the sums of 2 elements $\lambda_{1}=6 \lambda \pm 1, \lambda_{2}=6(v-\lambda) \pm 1$, respectively, over the remainders $m=(0,2,-2)$.

For any even numbers $\zeta>8$ on the segment $[1 \div v]$ there always exists a pair of numbers $\lambda_{1} \in\left[1 \div\left[\frac{v}{2}\right]\right]$ and $\lambda_{2} \in\left[\left[\frac{v}{2}\right] \div v\right]$ such that both $\left(\lambda_{1}, \lambda_{2}\right) \in \Pi_{T w} \cup\left(F N^{+} \Delta F N^{-}\right)$.

\section{REFERENCES}

1. Prashar K. Primzahlverteilung. Berlin, Springer, 1957. 527 p.

2. Crandall R., Pomerance C. Primes: A Computational Perspective. N. York, Springer-Verlag, 2001. 545 p.

3. Bukhshtab A. A. Teoriia chisel [Number theory]. Moscow, Prosveshchenie Publ., 1966. 384 p.

4. Chermidov S. I. O faktorizatsii natural'nykh chisel [On factorization of natural numbers]. Dialogi o nauke, 2011, no. 2, pp. 68-69.

5. Chermidov S. I. Raspredelenie prostykh chisel. Algoritm chisel-bliznetsov i ikh beskonechnost' [Distribution of prime numbers. Twin numbers algorithm and their infinity]. Politematicheskii setevoi elektronnyi nauchnyi zhurnal Kubanckogo gosudarstvennogo agrarnogo universiteta, 2015, no. 06 (110), pp. 414-436. Available at: http://ej.kubagro.ru/a/viewaut.asp?id=4701 (accessed: 12.12.2019).

6. Chermidov S. I. Raspredelenie prostykh i sostavnykh chisel i ikh algoritmicheskie prilozheniia [Distribution of prime and composite numbers and their algorithmic applications]. Vestnik Astrakhanskogo gosudarstvennogo tekhnicheskogo universiteta. Seriia: Upravlenie, vychislitel'naia tekhnika i informatika, 2017, no. 3, pp. 48-64. 


\title{
INFORMATION ABOUT THE AUTHOR
}

Chermidov Sergey Ivanovich - Russia, 350040, Krasnodar, Kuban State University, Competitor for a Scientific Degree of the Department of Applied Mathematics; chermidov.sergei@mail.ru.

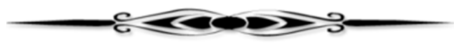

\section{ЗАКОН РАСПРЕДЕЛЕНИЯ ПРОСТЫХ ЧИСЕЛ. ЗАВИСИМОСТЬ ПРОСТЫХ ЧИСЕЛ ОТ ИХ ПОРЯДКОВЫХ НОМЕРОВ И БИНАРНАЯ ЗАДАЧА ГОЛЬДБАХА - ЭЙЛЕРА С ИСПОЛЬЗОВАНИЕМ ВЫЧИСЛИТЕЛЬНОЙ МАШИНЫ}

\author{
С. И. Чермидов \\ Кубанский государственньй университет, \\ Краснодар, Российская Федераичя
}

В статье рассматриваются методы определения и нахождения распределения составных чисел $C N$, простых чисел $P N$, двойников простых чисел $T w$ и двойников составных чисел $T w C N$, не имеющих делителей 2 и 3 в $\mathbb{N}$, основанные на множестве чисел типа $\Theta=\{6 \kappa \pm 1, \kappa \in \mathbb{N}\}$, являющемся полугруппой по отношению к умножению. Предложен метод получения простых чисел $p \geq 5$ по их порядковым номерам в множестве простых чисел $p \geq 5$ и наоборот, а также новый алгоритм поиска и распределения простых чисел на основе замкнутости элементов множества $\Theta$. В статье показано, что составное число $n \in \Theta$ представимо в виде произведений $(6 x \pm 1)(6 y \pm 1)$, где $x, y \in \mathbb{N}$ - целочисленные положительные решения одного из 4-х диофантовых уравнений: $P(x, y, \lambda)=6 x y \pm x \pm y-\lambda=0$. Доказано, что если существует параметр $\lambda$ двойников простых чисел, то ни одно из диофантовых уравнений $P(x, y, \lambda)=0$ не имеет положительных целых решений. Найден новый закон распределения простых чисел $\pi(x)$ в сегменте $[1 \div N]$. Любое четное число $\zeta>8$ сравнимо с одним из чисел $m=(0,2,-2)$, т. е. $\zeta \equiv m(\bmod 6)$. Согласно вышеупомянутым остаткам $m$, четные числа $\zeta>8$ делятся на 3 типа, и каждый вид имеет свой собственный способ представления сумм из 2-х элементов множества $\Theta$. Для любого четного числа $\zeta>8$ на сегменте $[1 \div v]$, где $v=(\zeta-\mathrm{m}) / 6$, есть параметр четного числа; доказано, что всегда существует пара чисел $\left(\lambda_{1}, \lambda_{2}\right) \in[1 \div v]$, являющихся элементами объединения множеств параметров двойников простых чисел $\Pi_{T w}$ и параметров переходных чисел $\Pi_{U P C}$, т. е. числа вида $6 \lambda \pm 1$ с одинаковым $\lambda$, если форма $6 \lambda-1$ является простым числом, то форма $6 \lambda+1$ является составным числом, и наоборот.

Ключевые слова: простые и составные числа, параметры простых чисел, диофантовы уравнения, бинарные (сильная) задача Гольдбаха - Эйлера, алгоритм решения бинарной задачи Гольдбаха - Эйлера.

Для цитирования: Чермидов С. И. Закон распределения простых чисел. Зависимость простых чисел от их порядковых номеров и бинарная задача Гольдбаха - Эйлера с использованием вычислительной машины // Вестник Астраханского государственного технического университета. Серия: Управление, вычислительная техника и информатика. 2020. № 4. C. 80-100. DOI: 10.24143/2072-9502-2020-4-80-100.

\section{СПИСОК ЛИТЕРАТУРЫ}

1. Prashar K. Primzahlverteilung. Berlin: Springer, 1957. 527 p.

2. Crandall R., Pomerance C. Primes: A Computational Perspective. N. York: Springer-Verlag, 2001. 545 p.

3. Бухштаб А. А. Теория чисел. М.: Просвещение, 1966. 384 с. 
4. Чермидов С. И. О факторизации натуральных чисел // Диалоги о науке. 2011. № 2. С. 68-69.

5. Чермидов С. И. Распределение простых чисел. Алгоритм чисел-близнецов и их бесконечность // Политемат. сетевой электр. науч. журн. Кубан. гос. аграр. ун-та. 2015. № 06 (110). C. 414-436. URL: http://ej.kubagro.ru/a/viewaut.asp?id=4701 (дата обращения: 12.12.2019).

6. Чермидов С. И. Распределение простых и составных чисел и их алгоритмические приложения // Вестн. Астрахан. гос. техн. ун-та. Сер.: Управление, вычислительная техника и информатика. 2017. № 3. С. $48-64$.

Статья поступила в редакцию 23.01.2020

\section{ИНФОРМАЦИЯ ОБ АВТОРЕ}

Чермидов Сергей Иванович - Россия, 350040, Краснодар; Кубанский государственный университет; соискатель кафедры прикладной математики; chermidov.sergei@mail.ru. 